\title{
Magic supergravities, $N=\mathbf{8}$ and black hole composites
}

\author{
Sergio Ferrara, ${ }^{1,2,3, *}$ Eric G. Gimon, ${ }^{4,5, \dagger}$ and Renata Kallosh ${ }^{6,+}$ \\ ${ }^{1}$ Physics Department, Theory Unit, CERN, 1211 Geneva 23, Switzerland INFN \\ ${ }^{2}$ Laboratori Nazionali di Frascati, Via Enrico Fermi, 40,00044 Frascati, Italy \\ ${ }^{3}$ Department of Physics and Astronomy, University of California, Los Angeles, California 90095, USA \\ ${ }^{4}$ Department of Physics, University of California, Berkeley, California 94720, USA \\ ${ }^{5}$ Theoretical Physics Group, LBNL, Berkeley, California 94720, USA \\ ${ }^{6}$ Physics Department, Stanford University, Stanford California 94305-4060, USA
}

(Received 14 July 2006; published 26 December 2006)

\begin{abstract}
We present explicit U-duality invariants for the $\mathbb{R}, \mathbb{C}, \mathbb{Q}, \mathbb{O}$ (real, complex, quaternionic, and octonionic) magic supergravities in four and five dimensions using complex forms with a reality condition. From these invariants we derive an explicit entropy function and corresponding stabilization equations which we use to exhibit stationary multicenter 1/2 Bogomol'nyi-Prasad-Sommerfield (BPS) solutions of these $N=2 d=4$ theories, starting with the octonionic one with $E_{7(-25)}$ duality symmetry. We generalize to stationary $1 / 8$ BPS multicenter solutions of $N=8, d=4$ supergravity, using the consistent truncation to the quaternionic magic $N=2$ supergravity. We present a general solution of nonBPS attractor equations of the STU truncation of magic models. We finish with a discussion of the BPSnon-BPS relations and attractors in $N=2$ versus $N=5,6,8$.
\end{abstract}

DOI: 10.1103/PhysRevD.74.125018

PACS numbers: 04.65.+e, 04.70.Bw

\section{INTRODUCTION}

The purpose of this paper is to present explicit stationary multicenter solutions of $N=8, d=4$ supergravity [1] and all magic $N=2$ supergravities [2]. All these models are associated with the Jordan algebras of $3 \times 3$ Hermitian matrices: $J_{3}^{\mathbb{R}}, J_{3}^{\mathbb{C}}, J_{3}^{\mathbb{Q}}, J_{3}^{\mathbb{Q}}$, and $J_{3}^{\mathbb{Q}_{s}}$. Here $\mathbb{A}=\mathbb{R}, \mathbb{C}, \mathbb{Q}, \mathbb{O}$ are the four division algebras, with $\operatorname{dim} \mathbb{A}=1,2,4,8$, while $\mathbb{O}_{s}$ is a the split form of $\mathbb{O}[3]$ with a quadratic norm invariant under $O(4,4)$ (the indefinite signature means this is no longer a division algebra). The octonionic magic $N=2$ model goes by the name exceptional since it is the only one of the four magic $N=2$ supergravities which is not known to be a consistent reduction of $N=8$ : its defining Jordan algebra $J_{3}^{\mathbb{Q}}$ involves the real octonions with a quadratic norm invariant under $O(8)$ [3], and is the only algebra mentioned above which one cannot get from truncating $J_{3}^{\mathbb{Q}_{s}}$. Note that the connection to Jordan algebras based on the division algebras defines a relation between the four $N=2$ magic supergravities [2] and the magic square [4]. From a physics perspective, the main feature of the $N=8$ and the magic $N=2$ supergravities which interests us is that for each case all the vector fields, including the graviphoton, transform in a single irreducible representation of U-duality group; these symmetries place strong constraints on the entropy formula and the stabilization equations derived from it.

The black hole entropy formula for $N=2$ supergravities based on symmetric spaces is either known or can be established. We extend this list to include magic super-

\footnotetext{
*Electronic address: Sergio.Ferrara@cern.ch

${ }^{\dagger}$ Electronic address: eggimon@lbl.gov

Electronic address: kallosh@stanford.edu
}

gravities, for which we construct these entropies. We can then give a complete list of all symmetric spaces with their entropy formulas.

The duality symmetry of $N=8, d=4$, and $d=5$ supergravities is $E_{7(7)}$ and $E_{6(6)}$, respectively. The duality symmetry of the exceptional magic $N=2, d=4$ and $d=$ 5 supergravities is $E_{7(-25)}$ and $E_{6(-26)}$, respectively. The number in the brackets stands for the difference between the number of noncompact minus compact generators. For example, in $E_{7(7)}$ and in $E_{7(-25)}$ the total number of noncompact and compact generators of $E_{7}$ is the same, namely, $70+63=133$ and $54+79=133$. However, the difference between them is either $70-63=7$ for $E_{7(7)}$ or $54-$ $79=-25$ for $E_{7(-25)}$.

For the octonionic $N=2$ supergravity we will find a new explicit entropy function derived from the quartic invariant of the fundamental 56-dimensional representation of $E_{7(-25)}$ and the relevant "electric" and "magnetic" cubic invariants of $E_{6(-26)}$. This entropy function then provide us with the most general explicit multicenter Bogomol'nyi-Prasad-Sommerfield (BPS) solution of the octonionic (exceptional magic) $N=2$ supergravity in terms of a 56-dimensional harmonic function with an arbitrary number of centers. We will also present explicit entropy formulas for all the remaining magic supergravities.

Recently, the generalization of the $N=2$ attractor equations in Refs. [5-8] was established for $N>2$ by a generalization of the special geometry symplectic structure to all extended supergravities [9]. In particular, for $N=8$ supergravity a simple set of algebraic equations was established which describe regular BPS and non-BPS extremal black hole solutions. In this later paper, a natural truncation of the $N=8$ theory to its largest consistent $N=2$ trunca- 
tion, the quaternion magic $N=2$ supergravity, made its appearance. To proceed with an explicit demonstration of stationary multicenter $1 / 8$ BPS solutions of $N=8 d=4$ supergravity we use this reduction to $N=2$. Hence, in addition to the $N=8$ model and the octonionic $N=2$ model, the $N=2$ magic supergravity based on the Jordan algebra of quaternions, $J_{3}^{\mathbb{Q}}$, is of particular interest in this paper.

We will argue that our new solution of quaternionic supergravity, depending on a 32-dimensional harmonic function with any number of centers, are also the most general $1 / 8$ BPS stationary multicenter solution of $N=8$ $d=4$ supergravity, up to an overall $E_{7(7)}$ rotation.

We also will analyze multiple relations between BPS and non-BPS solutions in $N=2$ and $N=5,6,8$ supergravities. It is known that the same bosonic solution may be BPS or non-BPS depending on how it is embedded in a given supergravity theory or how one identifies the bosonic vector fields with the specific supersymmetric multiplets, see, for example, Ref. [10] where many such examples were given. In some $N=8$ examples in Ref. [10] the relation between BPS and non-BPS solutions uses the same embedding but requires a flip of a sign from some of the charges. More recently such examples were discovered in the context of nonsupersymmetric attractors, see e.g. Ref. [11].

We will describe the general solutions of $N=2$ attractors and, in particular, the general solution for non-BPS attractors of magic supergravities.

In summary, our paper is organized as follows. In Sec. II we summarize and expand on known results for multicenter solutions in $N=2$ SUGRA with special emphasis on the role of the a set of harmonic functions transforming in an irreducible representation of the U-duality group. Writing down the metric, gauge and scalar fields depends crucially on a writing down a quartic invariant, or an entropy function, constructed from this representation. In section III we describe two approaches for writing down the quartic invariants for $N=8$ and the magic $N=2$ supergravities. The first approach uses connections to the five-dimensional U-duality group and makes novel use of a set of complex matrices with a reality condition in expressing both the entropy function and the attractor equations. The second approach stresses the connection of our quartic invariant to a set of invariants of the six-dimensional Uduality group which allows us to use real matrices (the quaternionic magic case is a pseudoreal exception). In Sec. IV we develop, as an example for the material in Secs. II and III, the solutions for BPS composites of octonionic magic $N=2$. Section V follows with a description of $N=81 / 8$ BPS composites in terms of a quaternionic $N=2$ subalgebra. In section VI we expand our circle of consideration to non-BPS extremal multicenter solutions before closing with some final thought in Sec. VII.

\section{A DESCRIPTION OF STATIONARY MULTICENTER SOLUTIONS: $N=2$ BLACK HOLE COMPOSITES}

The exact BPS multicenter stationary black hole solutions in $N=2$ supergravity have been worked out as a general case whenever there is a known expression for the explicit single center black hole entropy, $S(p, q)$, as a function of quantized charges [12]. The entropy formula is given by the minimal value of the BPS black hole mass via

$$
S(p, q)=\left.\pi M^{2}(p, q ; t, \bar{t})\right|_{\mathrm{attr}}=\pi I_{1}(p, q),
$$

where the moduli, $t(p, q), \bar{t}(p, q)$, are fixed near the black hole horizon by the attractor mechanism [5-8]. There are three invariants that the reader should be aware of in the context of black hole attractors, it is important not to confuse them:

(i) $I_{1}(p, q ; t, \vec{t})$ : This is the general symplectic invariant defined in $[6,13]$. It can be written explicitly as

$$
\begin{aligned}
I_{1}(p, q ; t, \bar{t}) & =-\frac{1}{2}(p q) \mathcal{M}(\mathcal{N}(t, \bar{t}))(p q)^{T} \\
& =|Z(\Gamma)|^{2}+|\mathcal{D} Z(\Gamma)|^{2},
\end{aligned}
$$

where $\mathcal{N}(t, \bar{t})$ is the metric on the vector fields. Here $Z(\Gamma)=\langle\Gamma, \Omega\rangle$ and $\Gamma=\left(p^{\Lambda}, q_{\Lambda}\right), \Omega=\left(L^{\Lambda}, M_{\Lambda}\right)$. This scalar quantity is an invariant of the group $\operatorname{OSp}\left(2\left(n_{v}+1\right), \mathbb{R}\right)$ which acts on both the charges and the symplectic sections. It is manifestly quadratic in the charges and non-negative. Near the black hole horizon $N=2$ supersymmetry is restored and $\mathcal{D Z}=0$ which is equivalent to the requirement that

$$
\partial_{t} I_{1}(\Gamma ; t, \bar{t})=0, \quad \partial_{\bar{t}} I_{1}(\Gamma ; t, \bar{t})=0 .
$$

This equation defines the moduli near the horizon as functions of charges, $t(p, q), \bar{t}(p, q)$.

(ii) $I_{1}(p, q)=I_{1}(p, q ; t(p, q), \bar{t}(p, q))$ : This is the entropy function, $I_{1}(\Gamma)$, derived from the function above restricted to $t$ 's and $\bar{t}$ 's implicitly defined in terms of $p$ 's and $q$ 's through their attractor values. This scalar is no longer invariant under $\operatorname{OSp}\left(2\left(n_{v}+\right.\right.$ $1), \mathbb{R})$ since we have chosen particular values of $t$ and $\bar{t}$ for given $p$ 's and $q$ 's. It is, however, invariant under the U-duality group if such exists, but it has no particular polynomial properties as a function of the charges. The moduli near the horizon are defined by equation [6]

$$
\begin{aligned}
& p^{\Sigma}+i \frac{\partial I_{1}(p, q)}{\partial q_{\Sigma}}=2 i \bar{Z}(\Gamma) L^{\Sigma}, \\
& q_{\Sigma}-i \frac{\partial I_{1}(p, q)}{\partial p^{\Sigma}}=2 i \bar{Z}(\Gamma) M_{\Sigma},
\end{aligned}
$$

which follows from $\mathcal{D} Z(\Gamma)=0$ at the horizon. The holomorphic special coordinates $t^{\Lambda}=\frac{X^{\Lambda}}{X^{0}}$ at the ho- 
rizon follow by dividing Eq. (2.4) on the zero component of the same equation.

$$
t^{\Lambda}(p, q)=\frac{p^{\Lambda}+i \frac{\partial I_{1}(\Gamma)}{\partial q_{\Lambda}}}{p^{0}+i \frac{\partial I_{1}(\Gamma)}{\partial q_{0}}} .
$$

(iii) $J_{4}(p, q)$ : This is a quartic polynomial in the charges, invariant under a U-duality group. It appears in all the theories discussed in this paper, it is related to the entropy function via $I_{1}(p, q)=\sqrt{\left|J_{4}(p, q)\right|}$.

Using the entropy function, the equations of motion for general black hole solutions in $N=2$ supergravities were solved in Refs. $[12,14]$. Examples of nonstatic multicenter solutions were given earlier in Ref. [15] where it was also explained on the basis of these examples that the most general expression for the metric can only depend on duality invariants, the Kähler potential $K(X, \bar{X})$ and the Kähler connection $\mathcal{A}_{\mu}(X, \bar{X})$. The general form of multicenter BPS black hole solutions was presented in Ref. [14].

The explicit BPS multicenter stationary black hole solutions in $N=2$ supergravity were found in Refs. $[12,14]$. One can solve the $1 / 2$ BPS equations by introducing a harmonic symplectic doublet:

$$
\mathbf{H}(\vec{x})=\left(H^{\Lambda}, H_{\Lambda}\right)=\mathbf{h}+\sum_{s=1}^{n} \frac{\boldsymbol{\Gamma}_{s}}{\left|\vec{x}-\vec{x}_{s}\right|} .
$$

From BPS equations one can prove that this doublet is proportional to the imaginary part of the covariantly holomorphic symplectic section $\Omega=\left(L^{\Lambda}, M_{\Lambda}\right)$ :

$$
\begin{aligned}
\mathbf{H}(\vec{x}) & =2 \operatorname{Im}\langle\mathbf{H}(\vec{x}), \bar{\Omega}\rangle \Omega \equiv i\left(H^{\Lambda} \bar{M}_{\Lambda}-H_{\Lambda} \bar{L}^{\Lambda}\right) \Omega, \\
\langle\Omega, \bar{\Omega}\rangle & =-i .
\end{aligned}
$$

We will refer to $\mathbf{h}$ and $\boldsymbol{\Gamma}$ as fundamentals since for the theories which appear in our paper they transform as fundamentals under the $E_{7} \mathrm{U}$-duality group. A symplectic invariant $I_{1}(\Gamma, t, \vec{t})$ is now replaced by the $\vec{x}$-dependent symplectic invariant $I_{1}(\mathbf{H}(\vec{x}), t, \vec{t})$. The other basic ingredient is the symplectic pairing of two charges which induces one on the harmonic functions:

$$
\left\langle\boldsymbol{\Gamma}_{1}, \boldsymbol{\Gamma}_{2}\right\rangle=p_{1}^{\Lambda} q_{2 \Lambda}-p_{2}^{\Lambda} q_{1 \Lambda} \hookrightarrow\left\langle\mathbf{H}_{1}, \mathbf{H}_{2}\right\rangle .
$$

Once equipped with these structures, one can prove that the BPS equations require that

$$
\langle H, \mathcal{D} \Omega\rangle=0,
$$

which results in

$$
\partial_{t} I_{1}(\mathbf{H}(\vec{x}) ; t, \vec{t})=0, \quad \partial_{\bar{t}} I_{1}(\mathbf{H}(\vec{x}) ; t, \vec{t})=0 .
$$

This is the stabilization equation for the symplectic invariant which can also be translated into a relation

$$
I_{1}\left(\left.(\mathbf{H}(\vec{x}) ; t, \vec{t})\right|_{\langle\mathbf{H}(\vec{x}), \mathcal{D} \Omega\rangle=0}=I_{1}((\mathbf{H}(\vec{x})) .\right.
$$

This leads to

$$
\begin{aligned}
& H^{\Sigma}+i \frac{\partial I_{1}(\mathbf{H})}{\partial H_{\Sigma}}=2 i \bar{Z}(\mathbf{H}) L^{\Sigma}, \\
& H_{\Sigma}-i \frac{\partial I_{1}(\mathbf{H})}{\partial H^{\Sigma}}=2 i \bar{Z}(\mathbf{H}) M_{\Sigma} .
\end{aligned}
$$

The special coordinates $t^{\Lambda}=\frac{X^{\Lambda}}{X^{0}}$ solve the equations of motion, stabilization equations of the same form as the attractor equations for moduli near the horizon, and are given by

$$
t^{\Lambda}(\vec{x})=\frac{H^{\Lambda}+i \frac{\partial I_{1}(H)}{\partial H_{\Lambda}}}{H^{0}+i \frac{\partial I_{1}(H)}{\partial H_{0}}} .
$$

The stationary metric is

$$
\begin{aligned}
d s_{4}^{2} & =-I_{1}^{-1}(\vec{x})(d t+\vec{\omega} d \vec{x})^{2}+I_{1}(\vec{x}) d \vec{x}^{2}, \\
\nabla \times \vec{\omega} & =\langle\mathbf{H}, \nabla \mathbf{H}\rangle .
\end{aligned}
$$

Note that $e^{-2 K(X, \bar{X})}$ may or may not be equal to $I_{1}(\vec{x})$, depending on the choice of the Kähler gauge. The symplectic invariant $I_{1}$ is invariant under Kähler transformations, $K(t, \bar{t}) \rightarrow K(t, \bar{t})+f(t)+\bar{f}(\bar{t})$, whereas the Kähler potential can be changed by a sum of the holomorphic and antiholomorphic function. In Ref. [14] an ansatz was used with $K=-2 U$ and $H=i \Omega_{0}-i \bar{\Omega}_{0}$, where $\Omega_{0}=$ $\left(X^{\Lambda}, F_{\Lambda}\right)$ is the holomorphic section. This section also transforms under Kähler transformations, $\Omega_{0} \rightarrow$ $\Omega_{0} e^{-f(t)}$. In Ref. [12] the ansatz for the harmonic doublet is $H=i e^{-U+K / 2}\left(e^{-i \alpha} \Omega_{0}-e^{i \alpha} \bar{\Omega}_{0}\right)$ where $\alpha$ is the argument of the central charge. We prefer to codify the solutions by the choice of the harmonic function at infinity, $\mathbf{h}$. Using this boundary value, we can give the values of the special coordinates, the metric and vector field strength and do not have to define combinations which are not invariant under Kähler transformations. For example, the relation between our $\mathbf{h}$ and the quantities in Ref. [12] is $\mathbf{h}=$ $\left(e^{-i \alpha} e^{K / 2} \Omega_{0}\right)_{\infty}$. In fact, only the product of all 3 terms is invariant under Kähler transformations. Therefore we can simply codify our solution with arbitrary $\mathbf{h}$. Note also that in our Eq. (2.11) the analogous term $\bar{Z}(\mathbf{H}) L^{\Sigma}$ is Kähler invariant since the transformations on $\bar{Z}(\mathbf{H})$ and on $L$ cancel and we do not have to identify these terms separately.

The integrability condition for the multicenter solution is

$$
\begin{aligned}
\langle\mathbf{H}, \triangle \mathbf{H}\rangle & =0 \Leftrightarrow\left\langle\mathbf{H}\left(\vec{x}_{s}\right), \boldsymbol{\Gamma}_{s}\right\rangle=0 \\
& \Leftrightarrow \sum_{t=1}^{n} \frac{\left\langle\boldsymbol{\Gamma}_{s}, \boldsymbol{\Gamma}_{t}\right\rangle}{\left|\vec{x}_{s}-\vec{x}_{t}\right|}+\left\langle\boldsymbol{\Gamma}_{s}, \mathbf{h}\right\rangle=0 .
\end{aligned}
$$

Hence when our generic set of $n+1$ fundamentals, $\left(\boldsymbol{\Gamma}_{s}, \mathbf{h}\right)$, satisfies the integrability conditions, we constrain the distances between the $\Gamma_{s}$ fundamentals, $\left|\vec{x}_{s}-\vec{x}_{t}\right|$. Only when two of these fundamental charge vectors are mutually local is their relative distance unconstrained. For the 
case when all the fundamentals are mutually local there are no constraints on the distances; they are just moduli.

The fundamental which sets the asymptotic behavior, $\mathbf{h}$, also must satisfy some conditions if the metric is to have the correct normalization and the integrability conditions are to be solved:

$$
I_{1}(\mathbf{h})=1, \quad\langle\mathbf{h}, \boldsymbol{\Gamma}\rangle=0, \quad\left(\boldsymbol{\Gamma}=\sum_{s} \boldsymbol{\Gamma}_{s}\right) .
$$

The vector fields in electric basis, using a mixture of spherical coordinates $\left(r_{s}, \theta_{s}, \phi_{s}\right)$ around each center $\vec{x}_{s}$, are

$$
A_{\Lambda}=\partial_{H^{\Lambda}}\left(\ln I_{1}(H)\right)(d t+\omega)-\sum_{s} \cos \theta_{s} d \phi_{s} \otimes \Gamma_{\Lambda} .
$$

The features of the geometry at very large $|\vec{x}| \gg\left|\vec{x}_{s}-\vec{x}_{t}\right|$ can be read off from the asymptotic form of the solution. In this approximation

$$
\mathbf{H}(\vec{x}) \approx \mathbf{h}+\frac{\boldsymbol{\Gamma}}{|\vec{x}|}, \quad \boldsymbol{\Gamma}=\sum_{s=1}^{n} \boldsymbol{\Gamma}_{s},
$$

and

$$
\vec{\omega} \approx \sum_{s<t}\left\langle\boldsymbol{\Gamma}_{s}, \boldsymbol{\Gamma}_{t}\right\rangle \vec{e}_{s t} \times \frac{\vec{x}}{|\vec{x}|}, \quad \vec{e}_{s t}=\frac{\vec{x}_{s}-\vec{x}_{t}}{\left|\vec{x}_{s}-\vec{x}_{t}\right|} .
$$

Far from the core, this looks like a spherically symmetric black hole (see Ref. [16] for more details):

$$
d s_{4}^{2}=-e^{2 U} d t^{2}+e^{-2 U} d \vec{x}^{2}, \quad e^{-2 U(\vec{x})}=I_{1}\left(\mathbf{h}+\frac{\boldsymbol{\Gamma}}{|\vec{x}|}\right),
$$

whose entropy is equal to

$$
S(\boldsymbol{\Gamma})=\pi I_{1}(\boldsymbol{\Gamma}) .
$$

Note that any measured angular momentum derived from our expansion of $\omega$ in (2.18) comes from terms which are order $1 /|\vec{x}|^{2}$ in the metric and should be matched with a measurement at a similar order for the dipole moments of the gauge fields in (2.16). Thus there is no contradiction with our knowledge that simple charged supersymmetric black holes have no angular momentum; if you measure angular momentum you also measure dipoles.

To recap, we would like to stress the special features of these $N=2$ solutions. For a solution with $n$ centers we needed $n+1$ constant fundamentals (symplectic doublets):

$$
\mathbf{h}=\left(h^{\Lambda}, h_{\lambda}\right), \quad \boldsymbol{\Gamma}_{s}=\left(p^{\Lambda}, q_{\Lambda}\right)_{s} \quad s=1, \ldots, n .
$$

giving these important qualities to our solutions:

(i) An attractor at infinity: This means that the values of all our special coordinates at infinity are specified by the values of the 1 st fundamental, $\mathbf{h}$ and are completely independent of all the other fundamentals,
$\boldsymbol{\Gamma}_{s}$. This follows from the limit of Eq. (2.12) at infinity.

$$
\left.t^{\Lambda}(\vec{x})\right|_{|\vec{x}| \rightarrow \infty} \rightarrow \frac{h^{\Lambda}+i \frac{\partial I_{1}(h)}{\partial h_{\Lambda}}}{h^{0}+i \frac{\partial I_{1}(h)}{\partial h_{0}}} .
$$

(ii) An attractor at each center: this is a familiar feature from BPS 1-center black holes where the values of the moduli near the black hole horizon are independent on the values of moduli at infinity. In our case, with many centers, the values of special coordinates at each center are independent of all fundamentals except the one at the given center. The limit of Eq. (2.12) near each center is given by

$$
\left.t^{\Lambda}(\vec{x})\right|_{|\vec{x}| \rightarrow\left|\vec{x}_{s}\right|} \rightarrow \frac{\Gamma_{s}^{\Lambda}+i \frac{\partial I_{1}\left(\Gamma_{s}\right)}{\partial \Gamma_{s \Lambda}}}{\Gamma_{s}^{0}+i \frac{\partial I_{1}\left(\Gamma_{s}\right)}{\partial \Gamma_{s 0}}} .
$$

Apart from the nice clarity of the attractor behavior above, at each center and at infinity, writing our solution in terms of $n+1$ fundamentals of our duality group yields two more simple features not yet discussed in the literature:

(i) The constant term in our integrability condition, (2.14), at each center is simply written in terms of the symplectic pairing between $\mathbf{h}$ and $\boldsymbol{\Gamma}_{\mathbf{s}}$ without any reference to the phase of the central charge such as in Ref. [12,14] (this simplification is also observed in Ref. [16]). This makes the construction, in principle, easier to extend to a non-BPS form.

(ii) The mass of the black hole in terms of the charges and asymptotic moduli can be written very compactly in terms of $\mathbf{h}$ and $\boldsymbol{\Gamma}$ by expanding $I_{1}(\vec{x})$ to leading order in $1 /|\vec{x}|$.

In what follows we will find that for most of the magic supergravities cases it is most convenient to generalize the form of the black hole composite solutions above: magic symmetries are best manifested using a double index, i.e. matrix, notation instead of a single index $\Lambda$ in coordinates and charges. We write real quantities, such as charges, as constrained complex matrices with a reality condition of type, $t^{*}=\Omega t \Omega^{T}$, and holomorphic quantities using the associated notion of complex conjugation.

\section{BLACK HOLE ENTROPIES OF MAGIC $N=\mathbf{2}$ AND OF $N=8$ SUPERGRAVITIES}

In order to find black hole composite solutions in four dimensions we require an explicit entropy formula in terms of generic black hole charges. Such formulas are known for symmetric spaces; the appropriate $N=2$ supergravity coupled to vector multiplets are classified in Refs. [1719]. These models include the reducible spaces $\left[\frac{S U(1,1)}{U(1)}\right]^{3}$, $\frac{S U(1,1)}{U(1)} \times \frac{S O(P+2,2)}{S O(P+2) \times S O(2)}$, and the complex projective space 
$\frac{S U(1, n)}{S O(n) \times U(1)}$. In Table 2 of Ref. [19] the first two cases are $L(0,0)$ and $L(0, P)$ respectively. For these three classes of $N=2 d=4$ supergravities the entropy formula is known for generic set of charges since the attractor equations [58] have been solved for these three spaces in Refs. [2022], respectively. The remaining symmetric spaces for which the entropy formulas have not been established so far are connected to Jordan algebras, according to Ref. [17]. These are the magic supergravities.

We will present here the new $d=4$ entropy formulas for magic $N=2$ supergravities using the relation between the $d=4, d=5$, and $d=6$ dualities. ${ }^{1}$ We will also give some new forms of the entropy formula for $N=8$ supergravity which can be easily compared with the entropy of the octonionic $N=2$.

\section{A. Universal $d=4 \Leftrightarrow d=5$ relation}

Consider the splitting

$$
G_{4} \rightarrow G_{5} \times S O(1,1),
$$

where $G_{5}$ is the duality group in $d=5$ and $G_{4}$ is the duality group in $d=4$. The quartic invariant of the relevant duality groups $G_{4}$ can be constructed using the cubic invariant of $G_{5}$. In all cases we have to split the total set of electric and magnetic charges into the zero component and the rest,

$$
(p, q)=\left(p^{0}, p^{I} ; q_{0}, q_{I}\right), \quad I=1, \ldots, n_{v} .
$$

This splitting is of the type

$$
\mathbf{R}_{p, q}=\mathbf{R}_{p}+\mathbf{1}_{p}+\mathbf{R}_{q}^{\prime}+\mathbf{1}_{q}^{\prime},
$$

where $\mathbf{R}_{p}$ is the five-dimensional representation and $\mathbf{R}_{q}^{\prime}$ is its contravariant representation:

$$
\begin{aligned}
\mathbf{R}_{p} \rightarrow\left(p^{I}\right), & \mathbf{R}_{q}^{\prime} \rightarrow\left(q_{I}\right), \\
\mathbf{1}_{p} \rightarrow\left(p^{0}\right), & \mathbf{1}_{q}^{\prime} \rightarrow\left(q_{0}\right) .
\end{aligned}
$$

All of these are real representations of $G_{4}$ or $G_{5}$ as appropriate. In the magic models $\mathbf{R}_{p, q}$ refers to the following representations:

$J_{3}^{\mathbb{Q}} \rightarrow \mathbf{5 6}, \quad$ fundamental of $E_{7(-25)}$,

$J_{3}^{\mathbb{Q}} \rightarrow 32, \quad$ chiral spinor of $S O^{*}(12)$,

$J_{3}^{\mathbb{C}} \rightarrow \mathbf{2 0}, \quad$ three fold antisymmetric (selfdual) of $S U(3,3)$,

$J_{3}^{\mathbb{R}} \rightarrow \mathbf{1 4}^{\prime}, \quad$ three fold antisymmetric traceless of $\operatorname{Sp}(6, \mathbb{R})$.

The general formula for the quartic invariant of the fourdimensional duality groups $G_{4}$ related to its $d=5$ cubic

\footnotetext{
${ }^{1}$ Recently the $d=4 \Leftrightarrow d=5$ relation between particular black hole solutions has been used in counting of the BPS black hole degeneracy [23].
}

invariants is

$$
\begin{aligned}
J_{4}\left(p^{0}, q_{0}, p^{I}, q_{I}\right)= & -(p \cdot q)^{2}+4\left(q_{0} I_{3}(p)-p^{0} I_{3}(q)\right. \\
& \left.+\left\{I_{3}(q), I_{3}(p)\right\}\right),
\end{aligned}
$$

where the cubic invariants of the five-dimensional duality groups $G_{5}$ are given by

$$
I_{3}(p)=\frac{1}{3 !} d_{I J K} p^{I} p^{J} p^{K}, \quad I_{3}(q)=\frac{1}{3 !} d^{I J K} q_{I} q_{J} q_{K},
$$

and the scalar product of charges and the Poisson bracket of cubic invariants are defined as follows:

$p \cdot q \equiv p^{0} q_{0}+p^{I} q_{I}, \quad\left\{I_{3}(q), I_{3}(p)\right\} \equiv \frac{\partial I_{3}(q)}{\partial q_{I}} \frac{\partial I_{3}(p)}{\partial p^{I}}$.

This structure of quartic invariants in terms of the cubic ones is valid for all cubic systems, where both $d_{I J K}$ as well as $d^{I J K}$ are known, in particular, it can be used for $N=8$ and for all the magic $N=2$ theories. Each model corresponds to specific values for $d_{I J K}$ and $d^{I J K}$ in Eq. (3.8) which form the cubic invariants of $G_{5}$. The quartic formula above can be derived following Refs. [3,24] using the Freudenthal triple system that applies for any decomposition of the type given in Eq. (3.1). The properties of the coset spaces which one encounters in magical supergravities can be inferred from Refs. [25,26].

For $N=8$ the classic example is $E_{7(7)} \rightarrow E_{6(6)} \times$ $S O(1,1)$. The quartic invariant of the $E_{7(7)}$ and the cubic invariant of the $E_{6(6)}$ have been in the literature a long time: they were used for the single black hole entropy in $N=8$, $d=4,[27,28]$ and $N=8, d=5$, [7], respectively. In this section, we will exploit the relationship between $N=8$ and the octonionic magic $N=2$ to efficiently present invariants for all the magic supergravities. We can use these, in turn, to write explicit black hole composite solutions.

Let us quickly review the magic $N=2$ supergravities. These are labeled using the four division algebras $\mathbb{A}$ : $\operatorname{dim} \mathbb{A}$ is 1 for real numbers $\mathbb{R}, 2$ for complex numbers $\mathbb{C}, 4$ for quaternions $\mathbb{H}$, and 8 for octonions $\mathbb{O}$. $N=8$ supergravity corresponds to the split octonions, which do not form a division algebra.

The scalars in $N=8$ supergravity are in the cosets $\frac{G_{5}}{H_{5}}=$ $\frac{E_{6(6)}}{U s p(8)}$ and $\frac{G_{4}}{H_{4}}=\frac{E_{7(7)}}{S U(8)}$ in $d=5$ and $d=4$, respectively. In each case, the vector fields are in the real representations 27 of $E_{6(6)}$ and $\mathbf{5 6}$ of $E_{7(7)}$.

For magic supergravities the cosets in $d=5$ are, in the order of increasing dimension of the division algebra $\operatorname{dim} A$ :

$$
d=5: \frac{G_{5}}{H_{5}} \Rightarrow \frac{S L(3 ; \mathbb{R})}{S O(3)}, \frac{S L(3 ; \mathbb{C})}{S U(3)}, \frac{S U^{*}(6)}{U s p(6)}, \frac{E_{6(-26)}}{F_{4}}
$$


and in four-dimensional theories

$$
\begin{aligned}
d=4: & \frac{G_{4}}{H_{4}} \Rightarrow \frac{S p(6 ; \mathbb{R})}{S U(3) \times U(1)}, \frac{S U(3,3)}{S U(3) \times S U(3) \times U(1)}, \\
& \frac{S O^{*}(12)}{S U(6) \times U(1)}, \frac{E_{7(-25)}}{E_{6} \times U(1)} .
\end{aligned}
$$

The number of vector fields in the five-dimensional versions of magic supergravities is

$$
\begin{aligned}
d & =5 \\
n_{v} & =3(\operatorname{dim} \mathbb{A}+1) \Rightarrow n_{v}=6,9,15,27 \text { for } \mathbb{R}, \mathbb{C}, \mathbb{H}, \mathbb{O},
\end{aligned}
$$

with one of the vectors coming from the gravity multiplet and transforming as a singlet of $H_{5}$. The "magic" comes from representing the charges for all these vector fields as real parameters of a self-adjoint $3 \times 3$ matrix over the appropriate algebra (elements of the Jordan algebra $J_{3}^{\mathbb{A}}$ ). In $d=4$, Kaluza-Klein reduction adds one more vector field, and so the numbers are

$$
\begin{aligned}
d & =4, \\
\tilde{n}_{v} & =3(\operatorname{dim} \mathbb{A}+1)+1 \Rightarrow \tilde{n}_{v} \\
& =7,10,16,28 \text { for } \mathbb{R}, \mathbb{C}, \mathbb{H}, \mathbb{O},
\end{aligned}
$$

with one of the vectors coming from the gravity multiplet and transforming as a singlet of $H_{4}$.

We will demonstrate two complementary methods for establishing the explicit form of the quartic/cubic invariants and related $d=4,5$ black hole entropies.

(1) Starting in five-dimensions, we use the manifest five-dimensional duality group $G_{5}$ for all of our models, with $E_{6(6)}, E_{6(-26)}, S U^{*}(6), S L(3 ; \mathbb{C})$, $S L(3 ; \mathbb{R})$-symmetry, and the simple features of the corresponding Jordan algebras to write down the appropriate cubic invariants. Operationally, this entails representing the real coordinates of the $N=8$ theory and the very special real geometry of the $N=$ 2 theories in terms of complex antisymmetric matrices obeying a reality condition. We then obtain the holomorphic coordinates of the corresponding four-dimensional scalar manifold geometry by relaxing the reality condition. To the reader, this may seem different from the well known case where one starts with real matrices in $d=5$ complexify them term by term to get the holomorphic coordinates in $d=4$. In fact, the difference is cosmetic rather than structural, due to the convenience of using a double index notation; we will make this clear in an explicit mathematical way.

(2) We rearrange our charges and coordinates using just $G_{6}$ manifestly-symmetric representations. In this case we have to further split the five-dimensional duality symmetry down to the six-dimensional one:

$$
G_{5} \Rightarrow G_{6} \times S O(1,1) .
$$

The duality group, $G_{6}$, for magic supergravities is $S O(1, \operatorname{dim} \mathbb{A}+1)$, similar to the duality group $S O(5,5)$ for the $N=8$ model. For a $I I B$ oxidation, tensor charges (self-dual strings) will appear in vector representations of $G_{6}$ and vector charges (point charges and their magnetic duals) will appear as real spinors. The advantage to this method is that, in all but one case, we can reconstruct the cubic invariant in $d=5$ using just the metric and gamma matrices of $G_{6}$. For the quaternionic magic the eight dimensional real representation of $S O(1,5)$ is really the combination of 2 pseudoreal spinors of the same chirality; the answer in this case is only slightly more complicated. In the first cases $(\operatorname{dim} \mathbb{A} \neq 4)$, the $d=4$ holomorphic coordinates are a simple complexification of the $d=6$ real coordinates. The details for the more subtle quaternionic case are presented in the appendix.

While overall not as manifestly symmetric, the sixdimensional approach provides us with more than a cross-check for our first method. The choice of splitting for $G_{5} \rightarrow G_{6} \times S O(1,1)$ also yields some valuable insight into the oxidation process, e.g. the split into tensor and vector multiplets above.

\section{B. $G_{5}$ manifestly-symmetric entropy of $d=4$ BPS black holes}

Our goal at this point is to develop a simple expression for the cubic invariants of the five-dimensional magic supergravity; using these we can then extract a $G_{5}$ manifestly-symmetric formula for the quartic invariant in four dimensions using the formula in Eq. (3.7). A very efficient way to accomplish our task is to use the relationship of these theories to Jordan algebras and the $N=8$ maximally supersymmetric theory.

Let us demonstrate with the familiar case of $N=8$ supergravity

$$
E_{7(7)} \rightarrow E_{6(6)} \times S O(1,1)
$$

and the octonionic magic $N=2$ supergravity with

$$
E_{7(-25)} \rightarrow E_{6(-26)} \times S O(1,1) .
$$

The respective five-dimensional charge spaces for these theories correspond to the Jordan algebras $J_{3}^{\mathbb{O}_{s}}$ and $J_{3}^{\mathbb{Q}}$. We can represent the 27 elements for both algebras conveniently using the (faithful) traceless antisymmetric representations of $U s p(8)$ and $U s p(6,2)$. This is similar in spirit to the use of $S U(8)$ and $S U(6,2)$ to construct quartic invariants in Ref. [29], but our results for the cubic invariants of $E_{6(6)}$ and $E_{6(-26)}$ do not descend straightforwardly from these quartic invariants. It would be interesting to have a better understanding of the connection between the two. 
We will now quickly review the details necessary to build these representations. We start by defining [25,26] the $(8 \times 8)$ symplectic matrix $\Omega$ and a metric preserved by $U s p(8)$ and $U s p(6,2)$

$$
\Omega=\mathbb{\square}_{4} \otimes\left(\begin{array}{cc}
0 & -1 \\
1 & 0
\end{array}\right), \quad K^{(a, b)}=\alpha \otimes\left(\begin{array}{ll}
1 & 0 \\
0 & 1
\end{array}\right),
$$

which will allow us to treat both cases, $E_{6(6)}$ in $U s p(8)$ basis and $E_{6(-26)}$ in $U s p(6,2)$ basis, simultaneously. The $(4 \times 4)$ matrix $\alpha$ is

$$
\begin{aligned}
\alpha & =\left(\begin{array}{llll}
1 & 0 & 0 & 0 \\
0 & 1 & 0 & 0 \\
0 & 0 & 1 & 0 \\
0 & 0 & 0 & 1
\end{array}\right) \text { for } \operatorname{Usp}(8), \\
\alpha & =\left(\begin{array}{cccc}
-1 & 0 & 0 & 0 \\
0 & -1 & 0 & 0 \\
0 & 0 & -1 & 0 \\
0 & 0 & 0 & 1
\end{array}\right) \text { for } \operatorname{Usp}(6,2),
\end{aligned}
$$

and $\alpha=\alpha^{t}, \alpha^{2}=1$. We will label the two different metrics either $K^{(0,4)}$ or $K^{(3,1)}$.

We are now ready to exhibit the real representation 27 of $E_{6(6)}, E_{6(-26)}$ taken in the $U s p(8), U s p(6,2)$ basis by using the map $t^{I} \rightarrow t^{a b}$ where $t^{a b}$ is the antisymmetric traceless matrix, $t^{a b}=-t^{b a}, \operatorname{Tr} t \equiv t^{a b} \Omega_{a b}=0$ satisfying a reality condition

$$
t_{a b}^{*}=\tilde{\Omega}_{a a^{\prime}} \tilde{\Omega}_{b b^{\prime}} t^{a^{\prime} b^{\prime}} \Leftrightarrow t^{*}=\tilde{\Omega} t \tilde{\Omega}^{T},
$$

where

$$
\begin{aligned}
& \tilde{\Omega}=\left(\begin{array}{cc}
0 & -\alpha \\
\alpha & 0
\end{array}\right)=K \Omega=\left(\begin{array}{ll}
\alpha & 0 \\
0 & \alpha
\end{array}\right)\left(\begin{array}{cc}
0 & -I \\
I & 0
\end{array}\right), \\
& \Omega=\left(\begin{array}{cc}
0 & -I \\
I & 0
\end{array}\right) .
\end{aligned}
$$

With our framework, we quickly write down our cubic invariant as

$$
d_{I J K} t^{I} t^{J} t^{K}=\operatorname{Tr}(\Omega t \Omega t \Omega t),
$$

which is a real polynomial. For the contragradient representation we can take

$$
d^{I J K} t_{I}^{\prime} t_{J}^{\prime} t_{K}^{\prime}=\operatorname{Tr}\left(\Omega^{\prime} t^{\prime} \Omega^{\prime} t^{\prime} \Omega^{\prime} t^{\prime}\right), \quad t^{\prime}=t_{a b}^{\prime}, \quad \Omega^{\prime}=\Omega^{\mathbf{T}} .
$$

Using these representations, the cubic invariants of $E_{6(6)}$ and $E_{6(-26)}$ in $U s p(8)$ and $U s p(6,2)$ basis, respectively, are given by

$$
\begin{aligned}
& I_{3}(p)=\frac{1}{3 !} \operatorname{Tr}(\Omega p \Omega p \Omega p), \\
& I_{3}(q)=\frac{1}{3 !} \operatorname{Tr}\left(\Omega^{\prime} q \Omega^{\prime} q \Omega^{\prime} q\right) .
\end{aligned}
$$

Here

$$
\begin{array}{ll}
p^{*}=\tilde{\Omega} p \tilde{\Omega}^{T}, & \operatorname{Tr}(p \Omega) \equiv p^{a b} \Omega_{a b}=0, \\
q^{*}=\tilde{\Omega} q \tilde{\Omega}^{T}, & \operatorname{Tr}(q \Omega)=0 .
\end{array}
$$

The reality and trace condition give exactly 27 real entries for the complex matrices $p^{a b}$ and $q_{a b}$; the complex two index notation is simply a convenient way to represent the real numbers $p^{I}$ and $q_{I}$. The only difference between the the cubic invariants of $E_{6(6)}$ and $E_{6(-26)}$ comes from the reality condition, driven by a different choice of $\tilde{\Omega}$ coming from different versions of the metric $K$. The the fact the cubic invariants are not explicitly different in terms of complex matrices, but only different through their respective reality constraint is a direct consequence of the fact that $E_{6(6)}$ and $E_{6(-26)}$ are different real forms of the same complex $E_{6}$ algebra.

The reality constraint (3.19) and the tracelessness condition $t^{a b} \Omega_{a b}=0$ can be solved and one finds that

$$
\begin{aligned}
& \operatorname{Re} t=\left(\begin{array}{cc}
A_{1} & S \\
-\alpha S \alpha & \alpha A_{1} \alpha
\end{array}\right), \\
& \operatorname{Im} t=\left(\begin{array}{cc}
A_{2} & A \\
\alpha A \alpha & -\alpha A_{2} \alpha
\end{array}\right),
\end{aligned}
$$

where $S^{t}=\alpha S \alpha, \operatorname{Tr} S=0$, and $A^{t}=-\alpha A \alpha$. This means that Ret depends on $n^{2}-1=15$ real entries since $A_{1}$ and $A_{2}$ and $A \alpha$ are antisymmetric, and $S \alpha$ is symmetric. $\operatorname{Im} t$ depends on $n^{2}-n=12$ real entries. Together $t$ has 27 real entries. The same forms apply for the electric and magnetic charges, in agreement with $n_{v}=3(\operatorname{dim} \mathbb{A}+1)=3(8+1)$ for octonions.

If we want to use only real entries for magnetic and electric charges, where $p=\operatorname{Re} p+i \operatorname{Im} p=p_{1}+i p_{2}$, we can use

$$
p^{*}=\tilde{\Omega} p \tilde{\Omega}^{T} \Rightarrow p_{1}=\tilde{\Omega} p_{1} \tilde{\Omega}^{T}, \quad p_{2}=-\tilde{\Omega} p_{2} \tilde{\Omega}^{T} .
$$

The cubic invariants in terms of the real entries are

$$
\begin{aligned}
I_{3}(p) & =\frac{1}{3 !} \operatorname{Tr}(\Omega p \Omega p \Omega p) \\
& =\frac{1}{3 !} \operatorname{Tr}\left(\Omega p_{1} \Omega p_{1} \Omega p_{1}\right)-\frac{1}{2} \operatorname{Tr}\left(\Omega p_{1} \Omega p_{2} \Omega p_{2}\right) .
\end{aligned}
$$

The electric charges are in the contragradient representation, and so we expand $q$ as $q=\operatorname{Re} q-i(-\operatorname{Im} q)=q_{1}-$ $i q_{2}$, with the analogous equations to Eqs. (3.26) and (3.27).

\section{Three other $G_{5}$-invariant magic entropies}

Consider the quaternionic magic case with $G_{5}=$ $S U^{*}(6), H_{5}=U s p(6)$. Our charges are in the 15, the two-fold antisymmetric representation $S U^{*}(6)$, which decomposes as the $\mathbf{1 4}$ traceless antisymmetric of $U s p(6)$ plus a singlet. This antisymmetric representation of $U s p(6)$ is not the truncation to the common $6 \times 6$ matrix of the two 
types of $8 \times 8$ matrices above (as opposed to what happens in Ref. [29]).

We write the quaternionic magic charges using the matrix $U^{\Lambda \Sigma}, U=-U^{t}$ with the reality condition $U^{*}=$ $\Omega U \Omega^{T}$, giving us $n_{v}=3(\operatorname{dim} \mathbb{A}+1)=3(4+1)=\frac{6 \cdot 5}{2}$, so we that we end up with 15 electric and 15 magnetic charges. The real cubic forms are

$$
\begin{aligned}
& \frac{1}{3 !} d_{I J K} t^{I} t^{J} t^{K}=\frac{1}{3 ! \cdot 2^{3}} \epsilon_{\Lambda \Sigma \Delta \Gamma \Pi \Omega} U^{\Lambda \Sigma} U^{\Delta \Gamma} U^{\Pi \Omega} \equiv \operatorname{Pf} U, \\
& I_{3}(p)=\frac{1}{3 ! \cdot 2^{3}} \epsilon_{\Lambda \Sigma \Delta \Gamma \Pi \Omega} p^{\Lambda \Sigma} p^{\Delta \Gamma} p^{\Pi \Omega} \equiv+\operatorname{Pf} p, \\
& I_{3}(q)=\frac{1}{3 ! \cdot 2^{3}} \epsilon^{\Lambda \Sigma \Delta \Gamma \Pi \Omega} q_{\Lambda \Sigma} q_{\Delta \Gamma} q_{\Pi \Omega} \equiv-\operatorname{Pf} q .
\end{aligned}
$$

In terms of real charges $p_{1}=\Omega p_{1} \Omega^{T}$ and $p_{2}=-\Omega p_{2} \Omega^{T}$ where $p^{*}=\Omega p \Omega^{T}$ and $p=p_{1}+i p_{2}$ we have

$$
I_{3}\left(p_{1}, p_{2}\right)=\frac{1}{3 ! \cdot 2^{3}} \epsilon_{\Lambda \Sigma \Delta \Gamma \Pi \Omega} p_{1}^{\Lambda \Sigma}\left(p_{1}^{\Delta \Gamma} p_{1}^{\Pi \Omega}-3 p_{2}^{\Delta \Gamma} p_{2}^{\Pi \Omega}\right)
$$

and analogous for $q=q_{1}-i q_{2}$. When we use these expressions (cf. Sec. V) to obtain a quartic invariant, we get an expression which matches exactly with the quartic invariant for quaternionic magic in Ref. [29]. This quartic invariant also appears explicitly in Ref. [30] within the context of $N=6$ supergravity.

The next case is complex magic with $G_{5} / H_{5}=$ $S L(3, \mathbb{C}) / S U(3)$ and $n_{v}=3(2+1)=9$. We truncate $U^{\Lambda \Sigma}$ by writing it in terms of a symmetric $3 \times 3$ matrix $U^{I \bar{I}}$ :

$$
U^{\Lambda \Sigma} \rightarrow U^{I \bar{I}}, \quad U_{\Lambda \Sigma} \rightarrow U_{I \bar{I}}
$$

The reality condition makes $U_{I \bar{I}}$ Hermitian (the trace is an invariant of $H_{5}$ ). The cubic invariants in term of real charges, where $p^{I \bar{I}}=\operatorname{Re} p^{I \bar{I}}+i \operatorname{Im} p^{I \bar{I}}=p_{1}^{I \bar{I}}+i p_{2}^{I \bar{I}}$ and $q_{I \bar{I}}=\operatorname{Re} q_{I \bar{I}}-i\left(-\operatorname{Im} q_{I \bar{I}}\right)=q_{1 \bar{I} \bar{I}}-i q_{2 I \bar{I}}$ are

$$
\begin{aligned}
I_{3}(p) & =\frac{1}{3 !} \epsilon_{I J K} \epsilon_{\bar{I} \bar{J} \bar{K}} p^{I \bar{I}} p^{J \bar{J}} p^{K \bar{K}}=\operatorname{det} p \\
& =\frac{1}{3 !} \epsilon_{I J K} \epsilon_{\bar{I} \bar{J} \bar{K}} p_{1}^{I \bar{I}}\left(p_{1}^{J \bar{J}} p_{1}^{K \bar{K}}-3 p_{2}^{J \bar{J}} p_{2}^{K \bar{K}}\right), \\
I_{3}(q) & =\frac{1}{3 !} \epsilon^{I J K} \epsilon^{\bar{I} \bar{J} \bar{K}} q_{I \bar{I}} q_{J \bar{J}} q_{K \bar{K}}=\operatorname{det} q \\
& =\frac{1}{3 !} \epsilon^{I J K} \epsilon^{\bar{I} \bar{J} \bar{K}} q_{1 I \bar{I}}\left(q_{1 J \bar{J}} q_{1 K \bar{K}}-3 q_{2 J \bar{J}} q_{2 K \bar{K}}\right) .
\end{aligned}
$$

The last case, real magic has $t^{I}$ in 6 of $S L(3, \mathbb{R})$, and $n_{v}=$ $3(1+1)=6$.

$$
\frac{1}{3 !} d_{I J K} t^{I} t^{J} t^{K}=\frac{1}{3 !} \epsilon_{L M N} \epsilon_{P Q R} t^{L P} t^{M Q} t^{N R}=\operatorname{det} t .
$$

Here the symmetric matrix $t^{L P}=t^{P L}$ is real. The cubic invariants in terms of real charges are

$$
\begin{aligned}
& I_{3}(p)=\frac{1}{3 !} \epsilon_{L M N} \epsilon_{P Q R} p^{L P} p^{M Q} p^{N R}, \\
& I_{3}(q)=\frac{1}{3 !} \epsilon^{L M N} \epsilon^{P Q R} q_{L P} q_{M Q} q_{N R} .
\end{aligned}
$$

\section{Magic prepotentials from $d=5$ and the attractor equations}

To connect the $d=5$ construction with real coordinates $Y^{I}$ to four-dimensional holomorphic coordinates $X^{I}$ we have to give a prescription for complexifying the real coordinates. We can then present the holomorphic prepotentials $F=\frac{I_{3}\left(X_{1}, X_{2}\right)}{X^{0}}$ of $d=4 N=2$ supergravities for all of our models. For the magic supergravities, the prescription is sweet and simple: we just relax the reality condition on our defining matrices, keeping it only when we need to define a complex conjugate quantity. In the quaternionic case, for example, we take our reality-constrained matrices $Y$ and split them once again into real matrices $Y=Y_{1}+$ $i Y_{2}$ satisfying $Y_{1}=\Omega Y_{1} \Omega^{T}, Y_{2}=-\Omega Y_{2} \Omega^{T}$. Regular complexification now requires us to turn the real matrices $Y_{1}, Y_{2}$ into complex matrices $X_{1}, X_{2}$ satisfying the linear constraint $X_{1}=\Omega X_{1} \Omega^{T}, X_{2}=-\Omega X_{2} \Omega^{T}$. If we write $X=X_{1}+i X_{2}$ we now have a new complex matrix independent of any linear constraints, the new conjugate variable is $\bar{X}=X_{1}^{*}+i X_{2}^{*}=\Omega\left(X^{*}\right) \Omega^{T}$.

To summarize, in general we apply the standard complexification to our real coordinates $Y_{1}$ and $Y_{2}$ to get a holomorphic coordinate $X=X_{1}+i X_{2}$. In the bi-index notation, what remains of the reality constraint on $Y$ is a different definition for complex conjugation on our vector space:

$$
\Rightarrow Y^{\Lambda \Sigma} \rightarrow X^{\Lambda \Sigma}, \quad \bar{X}^{\Lambda \Sigma}=\Omega\left(X^{\Lambda \Sigma}\right)^{*} \Omega^{T}
$$

Thus the "bar" operation is defined to be not just complex conjugation but also a matrix multiplication as shown above.

The prepotentials for all magic $N=2$ supergravities the their cubic invariant in five dimensions. These prepotentials may be used in future for the analysis of the Legendre transform and relation to the generalized Hitchin functional, as in Refs. [24,31].

$$
\begin{aligned}
J^{\mathbb{Q}} & \Rightarrow F(X) \\
& =\frac{1}{3 ! X^{0}} \operatorname{Tr}\left(\Omega\left(X_{1}+i X_{2}\right) \Omega\left(X_{1}+i X_{2}\right) \Omega\left(X_{1}+i X_{2}\right)\right) \\
& =\frac{1}{3 ! X^{0}}\left[\operatorname{Tr}\left(\Omega X_{1} \Omega X_{1} \Omega X_{1}\right)-3 \operatorname{Tr}\left(\Omega X_{1} \Omega X_{2} \Omega X_{2}\right)\right],
\end{aligned}
$$




$$
\begin{array}{ll}
X_{1}=\tilde{\Omega} X_{1} \tilde{\Omega}^{T}, & \operatorname{Tr}\left(X_{1} \Omega\right)=0, \\
X_{2}=-\tilde{\Omega} X_{2} \tilde{\Omega}^{T}, & \operatorname{Tr}\left(X_{2} \Omega\right)=0 .
\end{array}
$$

Here $X_{1}$ and $X_{2}$ are the complexified fields corresponding to the real and imaginary parts of the antisymmetric traceless matrix $Y^{a b}$ with $Y^{*}=-\tilde{\Omega} Y \tilde{\Omega}, a, b=1, \ldots, 8$.

$$
\begin{aligned}
J^{\mathbb{Q}} & \Rightarrow F(X)=\frac{1}{X^{0}} \operatorname{Pf}\left(X_{1}+i X_{2}\right) \\
& =\frac{1}{3 ! \cdot 2^{3} X^{0}} \epsilon_{\Lambda \Sigma \Delta \Gamma \Pi \Omega} X_{1}^{\Lambda \Sigma}\left(X_{1}^{\Delta \Gamma} X_{1}^{\Pi \Omega}-3 X_{2}^{\Delta \Gamma} X_{2}^{\Pi \Omega}\right) .
\end{aligned}
$$

Here $X_{1}$ and $X_{2}$ are antisymmetric and $X_{1}=\Omega X_{1} \Omega^{T}$, $X_{2}=-\Omega X_{2} \Omega^{T}$, with $\Lambda, \Sigma=1 \ldots 6$.

$$
\begin{aligned}
J^{\mathbb{C}} & \Rightarrow F(X)=\frac{1}{X^{0}} \operatorname{det}\left(X_{1}+i X_{2}\right) \\
& =\frac{1}{3 ! X^{0}} \epsilon_{I J K} \epsilon_{\bar{I} \bar{J} \bar{K}} X_{1}^{I \bar{I}}\left(X_{1}^{J \bar{J}} X_{1}^{K \bar{K}}-3 X_{2}^{J \bar{J}} X_{2}^{K \bar{K}}\right) .
\end{aligned}
$$

Here $X_{1}$ is symmetric and $X_{2}$ is antisymmetric. We can put them together in a single $3 \times 3$ matrix, $X$ in the $(\mathbf{3}, \overline{\mathbf{3}})$ representation of $H_{4}=S(U(3) \times U(3))$.

$$
J^{\mathbb{R}} \Rightarrow F(X)=\frac{1}{X^{0}} \operatorname{det} X=\frac{1}{3 ! X^{0}} \epsilon_{L M N} \epsilon_{P Q R} X^{L P} X^{M Q} X^{N R} .
$$

Here $X^{L M}$ is in the symmetric representation of $H_{4}=$ $U(3), L, M=1,2,3$.

In each case, the $X^{0}$ field is the usual extra projective coordinate which is a singlet of $H_{5}$. We note the parallel with the five-dimensional case. In that case, the real cubic invariant was determined by a cubic invariant of the complex form of $G_{5}$ plus a reality condition. Here the holomorphic prepotential is determined by a cubic invariant of $\mathrm{H}_{4}$ supplemented by a different notion of complex conjugation.

For completeness we add here the prepotentials for other $N=2$ cosets for which the entropy formula is known. For the $L(0, P)$ models the holomorphic prepotential is

$$
\begin{gathered}
F(X)=\frac{X}{2 X^{0}}\left[Y^{r} Y^{s} \eta_{r s}\right], \quad \eta_{r s}=(1,-1, \ldots,-1), \\
r, s,=1, \ldots, P+2 .
\end{gathered}
$$

The corresponding coset space in $D=4$ is $\frac{S U(1,1)}{U(1)} \times$ $\frac{S O(2, P+2)}{S O(2) \times S O(P+2)}$ and in $d=5$ we have $S O(1,1) \times \frac{S O(1, P+1)}{S O(P+1)}$ and the entropy was found in Ref. [21].

The case of the complex projective space $\frac{S U(1, n)}{S O(n) \times U(1)}$ is described by a quadratic prepotential

$$
F(X)=\frac{-i}{4}\left[\left(X^{0}\right)^{2}-\sum_{i=1}^{i=n}\left(X^{i}\right)^{2}\right]
$$

the entropy was found in Ref. [22].
The BPS attractor equations for all cases can be nicely written by once more embedding our charges into constrained complex matrices (here we use quaternionic magic to illustrate):

$$
p^{\Lambda \Sigma}+2 i \frac{\partial \sqrt{J_{4}(p, q)}}{\partial q_{\Lambda \Sigma}}=2 i \bar{Z} L^{\Lambda \Sigma} .
$$

In terms of strictly real charges $p^{1}, p^{2}$ and $q_{1}, q_{2}$ these equations can be rewritten as

$$
\begin{gathered}
p_{1}+i \frac{\partial \sqrt{I_{4}(p, q)}}{\partial q_{1}}=i \bar{Z}\left[L+\Omega L \Omega^{T}\right]=2 i \bar{Z} L_{1}, \\
p_{2}+i \frac{\partial \sqrt{I_{4}(p, q)}}{\partial q_{2}}=i \bar{Z}\left[L-\Omega L \Omega^{T}\right]=2 i \bar{Z} i L_{2} .
\end{gathered}
$$

Solving these attractor equations yields (in terms of either the constrained complex form or split real form of the charges):

$$
\begin{gathered}
t^{\Lambda \Sigma}=\frac{X^{\Lambda \Sigma}}{X^{0}} \rightarrow \frac{p^{\Lambda \Sigma}+2 i \frac{\partial \sqrt{J_{4}(p, q)}}{\partial q_{\Lambda \Sigma}}}{p^{0}+i \frac{\partial \sqrt{J_{4}(p, q)}}{\partial q_{0}}}, \\
\bar{t}^{\Lambda \Sigma}=\frac{\bar{X}^{\Lambda \Sigma}}{\bar{X}^{0}} \rightarrow \frac{p^{\Lambda \Sigma}-2 i \frac{\partial \sqrt{J_{4}(p, q)}}{\partial q_{\Lambda \Sigma}}}{p^{0}-i \frac{\partial \sqrt{J_{4}(p, q)}}{\partial q_{0}}}, \\
t_{1}^{\Lambda \Sigma}=\frac{X_{1}^{\Lambda \Sigma}}{X^{0}} \rightarrow \frac{p_{1}^{\Lambda \Sigma}+i \frac{\partial \sqrt{J_{4}(p, q)}}{\partial q_{1 \Lambda \Sigma}}}{p^{0}+i \frac{\partial \sqrt{J_{4}(p, q)}}{\partial q_{0}}}, \\
t_{2}^{\Lambda \Sigma}=\frac{X_{2}^{\Lambda \Sigma}}{X^{0}} \rightarrow \frac{p_{2}^{\Lambda \Sigma}+i \frac{\partial \sqrt{J_{4}(p, q)}}{\partial q_{2 \Lambda \Sigma}}}{p^{0}+i \frac{\partial \sqrt{J_{4}(p, q)}}{\partial q_{0}}},
\end{gathered}
$$

where we also exhibit the matching antiholomorphic equation to reemphasize the different complex conjugation. These equations are a slight generalization of the one in Eq. (2.12) which allow us accommodate a more algebraic notion of "reality" acting on matrices and will allow us to present the black hole composite solutions for $N=8$ and magic $N=2$ supergravities.

Finally, we can give an explicit expression for the Kähler potential of all the magic supergravities in terms of a modified notion of reality as follows. In standard holomorphic coordinates, the Kähler potential depends on the imaginary section. We then get the usual looking Kähler potential:

$$
e^{-K}=\frac{i}{3 !}\left|X^{0}\right|^{2} \operatorname{Tr}[\Omega(t-\bar{t}) \Omega(t-\bar{t}) \Omega(t-\bar{t})] .
$$

One has to keep in mind here that we use a definition of complex conjugation where our antiholomorphic special coordinates are related to the holomorphic as follows: 


$$
\bar{t}=\Omega^{T} t^{*} \Omega \text {. }
$$

This expression for the Kähler potential again shows that it is not invariant under Kähler transformations. One can use the gauge $X^{0}=1$, but in this gauge one does not necessarily have $2 U=K$. Moreover, it is known from the example of the STU attractor that in this gauge $e^{-2 U}$ is not equal to $e^{K}$, see Ref. [20]. On the other hand the freedom in the choice of the gauge for $X^{0}$ may be used to identify $K$ with $2 U$ as in Ref. [14].

\section{D. $G_{6}$ manifestly-symmetric entropy of $d=4 \mathrm{BPS}$ black holes}

The explicit expressions for each case can be established using the very special real geometry constructions of $[18,19]$. The corresponding homogeneous special real spaces in five dimensions (very special geometry) are

$$
\frac{G_{5}}{H_{5}}=L(P, 1), \quad P=\operatorname{dim} \mathbb{A}=1,2,4,8 .
$$

in the Table 2 of Ref. [19]. Their corresponding Kähler spaces with special geometry are our magic cosets $\frac{G_{4}}{H_{4}}$ in $d=4$. We uplift our models to $d=6(2,0)$ supergravities, following Refs. [32,33] (see also Ref. [34]). We introduce in $d=6$ a number of tensor multiplets, $N_{t}=\operatorname{dim} \mathbb{A}+1$, and a number of vector multiplets $N_{v}=2 \operatorname{dim} \mathbb{A}$ and we split the symmetric tensor $d_{I J K}$ as follows:

$$
d_{I J K} \Rightarrow\left(d_{z r s}, d_{r \alpha \beta}\right), \quad d^{I J K} \Rightarrow\left(d^{z r s}, d^{r \alpha \beta}\right),
$$

where $r=0, \ldots, \operatorname{dim} \mathbb{A}+1, z=1, \alpha, \beta=1, \ldots, 2 \operatorname{dim} \mathbb{A}$. This corresponds to the split of the real five-dimensional coordinates into $\left(z, b^{r}, a^{\alpha}\right)$ so that $z$ is the KK singlet scalar, $b^{r}$ is the coordinate of the coset space $\frac{S O(1, \operatorname{dim} \mathbb{A}+1)}{S O(\operatorname{dim} \mathbb{A}+1)}$ and $a^{\alpha}$ are spinors of $S O(1, \operatorname{dim} \mathbb{A}+1)$. We have an analogous split for the electric and magnetic charges.

$$
\begin{aligned}
& p^{I}=\left(p^{z}, p^{r}, p^{\alpha}\right), \quad q_{I}=\left(q_{z}, q_{r}, q_{\alpha}\right), \\
& r=0, \ldots, \operatorname{dim} \mathbb{A}+1, \quad \alpha=1, \ldots, 2 \operatorname{dim} \mathbb{A} .
\end{aligned}
$$

For the $L(0, P)$ models one should take $r, s=0, \ldots, P+1$ and $p^{\alpha}=q_{\alpha}=0$, following Table 2 of Ref. [19]. The cubic invariants are

$$
\begin{gathered}
I_{3}(p)=\frac{1}{2}\left(p^{z} \eta_{r s} p^{r} p^{s}+\left(\gamma_{r}\right)_{\alpha \beta} p^{r} p^{\alpha} p^{\beta}\right), \\
I_{3}(q)=\frac{1}{2}\left(q_{z} \eta^{r s} q_{r} q_{s}+\left(\gamma^{r}\right)^{\alpha \beta} q_{r} q_{\alpha} q_{\beta}\right) .
\end{gathered}
$$

Here $\eta_{r s}$ is the Lorentzian metric of $S O(1, \operatorname{dim} \mathbb{A}+1)$ and $\left(\gamma_{r}\right)_{\alpha \beta}$ are the $\gamma$-matrices of the groups $S O(1, \operatorname{dim} \mathbb{A}+1)$. The details magic quaternionic supergravity are given in the appendix. For the groups $S O(1,2), S O(1,3)$ and $S O(1,9)$ we can have real spinors and therefore the cubic invariants (3.55) and (3.56) above applies to them.

The relation between the magic exceptional octonionic $N=2$ and $N=8$ supergravity has to do with the change from the real octonions with the norm invariant under $S O(8)$ to the split octonions with the norm invariant under $S O(4.4)$. In $d=6$ this means that

$N=2 \quad$ octonionic magic, real octonions $\Rightarrow \frac{S O(1,9)}{S O(9)}$,

$N=8 \quad$ octonionic, split octonions $\Rightarrow \frac{S O(5,5)}{S O(5) \times S O(5)}$.

To find the cubic/quartic invariants of $N=8$ theory in the $G_{6}$ manifest basis all we have to do it to take the cubic invariants for octonionic magic $N=2$ in Eqs. (3.55) and (3.56) with the associated metric $\eta_{r s}$ for $S O(5,5)$ instead of that for $S O(1,9)$. The $\gamma$-matrices in the second term of cubic invariants change accordingly so that the corresponding Clifford algebra has the $\eta_{r s}$ signature for $S O(5,5)$ instead of $S O(1.9)$.

The case of quaternionic magic supergravity with $S O(1,5) \sim S U^{*}(4)$ duality group we have to treat separately. We present this case in the appendix.

\section{BPS COMPOSITES OF OCTONIONIC MAGIC $N=\mathbf{2}$}

\section{A. Manifest $\boldsymbol{E}_{6(-26)}$}

The quartic invariant of $E_{7(7)}$ is a well known invariant related to the entropy of $N=8$ black holes. We would like to give a similar explicit formula for the entropy of the octonionic magic $N=2$ which is a quartic invariant of $E_{7(-25)}$. In this section we will extract this invariant using the procedure given in Sec. III, but in more detail. We will then use this expression to write down the general multiblack hole solution following the framework in Sec. II, with all the modifications necessary to use our the bi-index formalism which proved so useful in deriving the cubic and quartic invariants of octonionic magic.

Recapping the procedure in Sec. III, we introduce the charges with a $d=4 \rightarrow d=5$ split corresponding to the $E_{7(-25)} \rightarrow E_{6(-26)} \times S O(1,1)$ split; in the $N=8$ case this is an $E_{7(7)} \rightarrow E_{6(6)} \times S O(1,1)$ split. The real $\mathbf{5 6}$ of $E_{7(-25)}$ which combines electric and magnetic charges is split into 2 real charges $p^{0}, q_{0}$ and 54 electric and magnetic charges in the $27^{\prime}$ and 27 representations of $E_{6(-26)}$, written as representations of $U s p(6,2)$. This means we have a complex antisymmetric skew-traceless $p^{a b}=\left(p_{1}^{a b}+i p_{2}^{a b}\right)$ and $q_{a b}=\left(q_{1 a b}-i q_{2 a b}\right)$ satisfying a reality constraint $p^{*}=\tilde{\Omega} p \tilde{\Omega}^{T}$ and analogous for $q$. Here

$$
\tilde{\Omega}=K^{(3,1)} \Omega,
$$

where the matrices $K^{(3,1)}$ and $\Omega$ are defined in Eqs. (3.17) and (3.18). Alternatively, one could use the real charges, 
$p_{1}^{a b}, p_{2}^{a b}$ and $q_{1 a b}, q_{2 a b}$, as is and lose explicit duality symmetry.

The quartic invariants of $E_{7(7)}$ and $E_{7(-25)}$ are both

$$
\begin{aligned}
J_{4}\left(p^{0}, q_{0}, p^{a b}, q_{a b}\right)= & -(p \cdot q)^{2}+4\left(q_{0} I_{3}(p)-p^{0} I_{3}(q)\right. \\
& \left.+\left\{I_{3}(q), I_{3}(p)\right\}\right)
\end{aligned}
$$

with cubic invariants given by

$$
\begin{aligned}
I_{3}(p) & =\frac{1}{3 !} \operatorname{Tr}(\Omega p \Omega p \Omega p) \\
& =\frac{1}{3 !} \operatorname{Tr}\left(\Omega p_{1} \Omega p_{1} \Omega p_{1}\right)-\frac{1}{2} \operatorname{Tr}\left(\Omega p_{1} \Omega p_{2} \Omega p_{2}\right),
\end{aligned}
$$

$$
\begin{aligned}
I_{3}(q) & =\frac{1}{3 !} \operatorname{Tr}\left(\Omega^{\prime} q \Omega^{\prime} q \Omega^{\prime} q\right) \\
& =\frac{1}{3 !} \operatorname{Tr}\left(\Omega^{\prime} q_{1} \Omega^{\prime} q_{1} \Omega^{\prime} q_{1}\right)-\frac{1}{2} \operatorname{Tr}\left(\Omega^{\prime} q_{1} \Omega^{\prime} q_{2} \Omega^{\prime} q_{2}\right),
\end{aligned}
$$

and the scalar product of charges and the Poisson bracket of cubic invariants are defined as follows:

$$
\begin{aligned}
p \cdot q \equiv & p^{0} q_{0}+\frac{1}{2} p^{a b} q_{a b} \\
= & p^{0} q_{0}+\frac{1}{2} p_{1}^{a b} q_{1 a b}+\frac{1}{2} p_{2}^{a b} q_{2 a b}, \\
\left\{I_{3}(q), I_{3}(p)\right\} & \equiv 2 \frac{\partial I_{3}(q)}{\partial q_{a b}} \frac{\partial I_{3}(p)}{\partial p^{a b}} \\
& =\frac{1}{2} \frac{\partial I_{3}(q)}{\partial q_{1 a b}} \frac{\partial I_{3}(p)}{\partial p_{1}^{a b}}+\frac{1}{2} \frac{\partial I_{3}(q)}{\partial q_{2 a b}} \frac{\partial I_{3}(p)}{\partial p_{2}^{a b}} .
\end{aligned}
$$

All the formulas above with complex matrices are real due to the reality condition on the charge matrices. We note that any derivatives we write in the octonionic case need to take into account the fact that we differentiate with respect to skew-traceless matrices. This corresponds to restricting the tangent space using the tracelessness condition and operationally gives the following definition for the derivative:

$$
\frac{\partial p^{a b}}{\partial p^{c d}}=\delta_{c}^{a} \delta_{d}^{b}-\delta_{d}^{a} \delta_{c}^{b}-\frac{1}{4} \Omega_{a b} \Omega_{c d} .
$$

It would be interesting to understand exactly witch change of variable would be necessary to match with our expression for the quartic invariant, $J_{4}$, with the one in Ref. [29] written explicitly in terms of four-dimensional charges.

Overall, the only difference between the endpoint expressions for $E_{7(7)}$ and $E_{7(-25)}$ is exactly in the reality constraint on the charges, i.e. which parts of $p$ get slotted in $p_{1}$ or $p_{2}$ by our choice of $\tilde{\Omega}$ :

$$
\begin{aligned}
N & =8: E_{7(7)} \rightarrow E_{6(6)} \times S O(1,1) \Rightarrow p= \pm \tilde{\Omega}^{T} p^{*} \tilde{\Omega} \\
& =K^{0,4} \Omega^{T} p^{*} \Omega K^{0,4}, \\
N & =2 \text { magic: } E_{7(-25)} \rightarrow E_{6(-26)} \times S O(1,1) \Rightarrow p \\
& =\tilde{\Omega}^{T} p^{*} \tilde{\Omega}= \pm K^{3,1} \Omega^{T} p^{*} \Omega K^{3,1} .
\end{aligned}
$$

Following Sec. II, to describe the BPS composites of octonionic magic $N=2$ we need a set of $1+n$ fundamental 56-dimensional representations of $E_{7(-25)}$. Here $n$ is the number of centers of our multicenter solution

$$
\begin{gathered}
\mathbf{h} \equiv\left(h^{0}, h_{0}, h^{a b}, h_{a b}\right), \quad \boldsymbol{\Gamma}_{\mathbf{s}} \equiv\left(p^{0}, q_{0}, p^{a b}, q_{a b}\right)_{s}, \\
a, b=1, \ldots, 8, \quad s=1, \ldots, n .
\end{gathered}
$$

We introduce a 56-real-dimensional harmonic function in terms of two real harmonics and two constrained complex matrix harmonics:

$$
\mathbf{H}(\vec{x})=\left(H^{0}, H_{0}, H^{a b}, H_{a b}\right)=\mathbf{h}+\sum_{s=1}^{n} \frac{\boldsymbol{\Gamma}_{s}}{\left|\vec{x}-\vec{x}_{s}\right|} .
$$

Here we have introduced $n$ constant $E_{7(-25)}$ fundamentals $\boldsymbol{\Gamma}_{s} \equiv(p, q)_{s}$ with $s=1, \ldots, n$ and one constant $E_{7(-25)}$ fundamental, $h \equiv\left(p_{\infty}, q_{\infty}\right)$ which is the asymptotic value of the harmonic function. Note that once again reality for $\mathbf{H}$ in this notation means that $\left(H^{a b}, H_{a b}\right)$ are complex matrices, they satisfy the same reality condition as the corresponding charge matrices. With the information above, we are almost ready to write down the solution. The final ingredient we need is a definition of the symplectic invariant for our solution, which is

$$
\left\langle\boldsymbol{\Gamma}_{s}, \boldsymbol{\Gamma}_{t}\right\rangle=p_{s} \cdot q_{t}-p_{t} \cdot q_{s} .
$$

We can now write the stationary metric for the BPS multicenter solution with $J_{4}(\vec{x})>0$ :

$$
d s_{4}^{2}=-J_{4}^{-1 / 2}(\vec{x})(d t+\vec{\omega} d \vec{x})^{2}+J_{4}^{1 / 2}(\vec{x}) d \vec{x}^{2},
$$

where

$$
J_{4}(\vec{x}) \equiv J_{4} \circ \mathbf{H}(\vec{x}), \quad \nabla \times \vec{\omega}=\langle\mathbf{H}, \nabla \mathbf{H}\rangle .
$$

As before, we define $J_{4} \circ \mathbf{H}(\vec{x})$ by replacing the set of charges in Eqs. (4.2) by the harmonic functions in Eq. (4.11). The integrability condition for the solution is

$$
\langle\mathbf{H}, \triangle \mathbf{H}\rangle=0, \quad \sum_{t=1}^{n} \frac{\left\langle\boldsymbol{\Gamma}_{s}, \boldsymbol{\Gamma}_{t}\right\rangle}{\left|\vec{x}_{s}-\vec{x}_{t}\right|}+\left\langle\boldsymbol{\Gamma}_{s}, \mathbf{h}\right\rangle=0 .
$$

For the asymptotically flat geometry one has to require that $J_{4}(h)=1$. The values of the vector fields (written as constrained complex matrices again), in spherical coordinates $\left(r_{s}, \theta_{s}, \phi_{s}\right)$ around each center $\vec{x}_{s}$ are

$$
A^{a b}=\frac{\partial}{\partial H_{a b}}\left(\ln J_{4}(H)\right)(d t+\omega)-\sum_{s} \cos \theta_{s} d \phi_{s} \otimes \Gamma_{s}^{a b} .
$$


The 54 scalars of magic octonionic model represent the coset space $\frac{E_{7(-25)}}{E_{6} \times S O(2)}$. We give their solution in terms of the holomorphic coordinates with the special complex conjugation explained in Sec. III C:

$$
\frac{X^{a b}}{X^{0}}(\vec{x})=\frac{H^{a b}+2 i \frac{\partial \sqrt{J_{4}(H)}}{\partial H_{a b}}}{H^{0}+i \frac{\partial \sqrt{J_{4}(H)}}{\partial H_{0}}}
$$

If we want to write things in terms a purely real decomposition of $\mathbf{H}, H^{a b}=H_{1}^{a b}+i H_{2}^{a b}, H_{a b}=H_{1}^{a b}-i H_{2}^{a b}$ with $H_{1}=H_{1}^{*}=\tilde{\Omega} H_{1} \tilde{\Omega}^{T}$ and $H_{2}=H_{2}^{*}=-\tilde{\Omega} H_{2} \tilde{\Omega}^{T}$ we can split the equation above into two parts involving only real harmonic functions and two separate holomorphic functions $t_{1}=\tilde{\Omega} t_{1} \tilde{\Omega}^{T}$ and $t_{2}=-\tilde{\Omega} t_{2} \tilde{\Omega}_{2}$ :

$$
\begin{aligned}
& t_{1}^{\Lambda \Sigma}=\frac{X_{1}^{\Lambda \Sigma}}{X^{0}} \rightarrow \frac{H_{1}^{\Lambda \Sigma}+i \frac{\partial \sqrt{J_{4}(H)}}{\partial H_{1 \Lambda \Sigma}}}{H^{0}+i \frac{\partial \sqrt{J_{4}(H)}}{\partial H_{0}}}, \\
& t_{2}^{\Lambda \Sigma}=\frac{X_{2}^{\Lambda \Sigma}}{X^{0}} \rightarrow \frac{H_{2}^{\Lambda \Sigma}+i \frac{\partial \sqrt{J_{4}(H)}}{\partial H_{\Lambda \Sigma}}}{H^{0}+i \frac{\partial \sqrt{J_{4}(H)}}{\partial H_{0}}} .
\end{aligned}
$$

This completes the multicenter composite solution of the magic octonionic supergravity.

\section{B. Manifest $S O(1,9)$}

By uplifting the magic octonionic supergravity to $d=6$ and breaking $E_{6(-26)} \rightarrow S O(1,9) \times S O(1,1)$ we can present the entropy formula using only real unconstrained charges. The set of 27 real $d=5$ coordinates includes a singlet of $S O(1,9) z$, a 10-component vector $b^{r}$, and a chiral Majorana-Weyl 16-component spinor $\psi^{\alpha}$. The quartic invariant of $E_{7(-25)}$ in this basis can be presented as a function of real 56 charges $\left(p^{0}, p^{I} ; q_{0}, q_{I}\right)$ where

$$
p^{I}=\left(p^{z}, p^{r}, p^{\alpha}\right), \quad q_{I}=\left(q_{z}, q_{r}, q_{\alpha}\right) .
$$

Here $p^{\alpha}$ are $\mathbf{1 6}_{L}$ Majorana-Weyl spinors of $S O(1,9)$ and $q_{\alpha}$ are $\mathbf{1 6}_{R}$ Majorana-Weyl spinors of $S O(1,9)$.

$$
\begin{aligned}
J_{4}\left(p^{0}, p^{I}, q_{0}, q_{I}\right)= & -(p \cdot q)^{2}+4\left(q_{0} I_{3}(p)-p^{0} I_{3}(q)\right. \\
& \left.+\left\{I_{3}(q), I_{3}(p)\right\}\right),
\end{aligned}
$$

where the cubic invariants of the $E_{6(-26)}$ in this basis are given by

$$
\begin{aligned}
& I_{3}\left(p^{I}\right)=\frac{1}{2}\left(p^{z} \eta_{r s} p^{r} p^{s}+\left(\gamma_{r}\right)_{\alpha \beta} p^{r} p^{\alpha} p^{\beta}\right), \\
& I_{3}\left(q_{I}\right)=\frac{1}{2}\left(q_{z} \eta^{r s} q_{r} q_{s}+\left(\gamma^{r}\right)^{\alpha \beta} q_{r} q_{\alpha} q_{\beta}\right),
\end{aligned}
$$

and the scalar product of charges and the Poisson bracket of cubic invariants are defined as follows

$$
\begin{aligned}
p \cdot q & \equiv p^{0} q_{0}+p^{z} q_{z}+p^{r} q_{r}+p^{\alpha} q_{\alpha}, \\
\left\{I_{3}(q), I_{3}(p)\right\} & \equiv \frac{\partial I_{3}(q)}{\partial q_{I}} \frac{\partial I_{3}(p)}{\partial p^{I}} .
\end{aligned}
$$

Here $\eta_{r s}$ is the Lorentzian metric for the space $S O(1,9)$ and $\gamma$ are the chiral $\gamma$-matrices.

The relation between the entropy of the magic exceptional octonionic $N=2$ and $N=8$ supergravity in this basis is simple. The metric $\eta_{r s}$ for $N=8$ is that of $S O(5,5)$ instead of $S O(1,9)$ and the $\gamma$-matrices in the second term of cubic invariants are the one for $S O(5,5)$ instead of $S O(1,9)$.

Since the entropy of exceptional magic $N=2$ supergravity in Eqs. (4.20), (4.21), and (4.22) in $d=6$ basis is given in terms of the real 56 charges we may immediately use it for the standard from of the composite multicenter black hole solutions using equations from the Sec. II.

The choice of the most appropriate basis for the solutions, the one with manifest $E_{6(-26)}$ or with manifest $S O(1.9)$ may depend on the problem.

\section{V. $N=8$ BPS COMPOSITES}

\section{A. Solutions via truncation to quaternionic magic $N=2$}

$N=8$ supergravity with its $\frac{E_{7(7)}}{S U(8)}$ coset space can be consistently truncated to $N=2$ quaternionic magic supergravity with a $\frac{S O^{*}(12)}{U(6)}$ coset space [2]. Hence $1 / 2$ BPS multicenter solutions of quaternionic magic supergravity are simultaneously $1 / 8$ BPS multicenter solutions of $N=$ $8 d=4$ supergravity. This fact has the useful consequence that to generate multicenter solutions of $N=8$ we can use magic $N=2$ and the procedure summarized in the Sec. II to extend the black hole attractor equations to stabilization equations in terms of harmonic functions.

Our first step is to identify the proper set of 32 charges which transforms as a real spinor representation of the $S O^{*}(12)$ duality group and are truncated from the 56dimensional fundamental representation of the $E_{7(7)}$ duality group of the $N=8$ model. Note that $E_{7(7)}$ decomposes into $S U(2) \times S O^{*}(12)$ and therefore we can use the following split

$$
E_{7(7)} \rightarrow S U(2) \times S O^{*}(12), \quad \mathbf{5 6} \rightarrow(\mathbf{2}, \mathbf{1 2})+(\mathbf{1}, \mathbf{3 2}) .
$$

Thus we keep all 32 charges of $S O^{*}(12)$ and take as vanishing the remaining 24 charges of $E_{7(7)}$. For this quaternionic magic model we can use the formulas in Sec. III to write our expressions with the $G_{5}=S U^{*}(6)$ duality manifest or with the $G_{6}=S O(1,5) \sim S U^{*}(4)$ manifest. In both cases we naturally work with complex coordinates in $d=5$ satisfying a reality constraint. We will quickly recap here the solution with manifest $G_{5}=S O^{*}(12)$ dual- 
ity, and then discuss some interesting features of the solution obtained from the truncated $N=8$ structure. by

One 32-dimensional set of charges of $S O^{*}(12)$ is given

$$
\Gamma=\left(p^{0}, p^{\Lambda \Sigma} ; q_{0}, q_{\Lambda \Sigma}\right), \quad \Lambda, \Sigma=1, \ldots 6,
$$

where the $15 p^{\Lambda \Sigma}$ 's satisfy the constraint $p=\Omega p \Omega^{T}$ and similarly for the electric charges $q_{\Lambda \Sigma}$. These charges transform as an antisymmetric plus singlet of the compact subgroup $H_{5}=U s p(6)$. As in the octonionic case, we can split our charges as $p^{\Lambda \Sigma}=p_{1}^{\Lambda \Sigma}+i p_{2}^{\Lambda \Sigma}$ and $q_{\Lambda \Sigma}=$ $q_{1 \Lambda \Sigma}-i q_{2 \Lambda \Sigma}$. The cubic invariants are

$$
I_{3}(p)=+\operatorname{Pf} p, \quad I_{3}(q)=-\operatorname{Pf} q,
$$

and the scalar product of charges and the Poisson bracket of cubic invariants are defined as follows:

$$
\begin{gathered}
p \cdot q \equiv p^{0} q_{0}+\frac{1}{2} p^{\Lambda \Sigma} q_{\Lambda \Sigma} \\
=p^{0} q_{0}+\frac{1}{2} p_{1}^{\Lambda \Sigma} q_{1 \Lambda \Sigma}+\frac{1}{2} p_{2}^{\Lambda \Sigma} q_{2 \Lambda \Sigma} \\
\left\{I_{3}(q), I_{3}(p)\right\} \equiv 2 \frac{\partial I_{3}(q)}{\partial q_{\Lambda \Sigma}} \frac{\partial I_{3}(p)}{\partial p^{\Lambda \Sigma}} \\
=\frac{1}{2} \frac{\partial I_{3}(q)}{\partial q_{1 \Lambda \Sigma}} \frac{\partial I_{3}(p)}{\partial p_{1}^{\Lambda \Sigma}}+\frac{1}{2} \frac{\partial I_{3}(q)}{\partial q_{2 \Lambda \Sigma}} \frac{\partial I_{3}(p)}{\partial p_{2}^{\Lambda \Sigma}} .
\end{gathered}
$$

allowing us to define $J_{4}\left(p^{0}, q_{0}, p^{\Lambda \Sigma}, q_{\Lambda \Sigma}\right)$ (if we map $p^{0}$ and $q_{0}$ to $Z^{78}$ and $Z_{78}$ in Ref. [29] we get a matching expression for $J_{4}$ ).

The quaternionic multicenter solution starts with $(1+$ $n$ ) of these spinor representations of $S O^{*}(12)$. Here $n$ is the number of centers of our multicenter solution

$$
\begin{gathered}
\mathbf{h} \equiv\left(h^{0}, h^{\Lambda \Sigma} ; h_{0}, h_{\Lambda \Sigma}\right), \quad \boldsymbol{\Gamma}_{s} \equiv\left(p^{0}, p^{\Lambda \Sigma} ; q_{0}, q_{\Lambda \Sigma}\right)_{s}, \\
s=1, \ldots, n .
\end{gathered}
$$

The first set $\mathbf{h}$ defines the value of the harmonic spinor at infinity. The other $n$ spinors are the quantized electric and magnetic charges at each center. We can now introduce a constrained complex harmonic function:

$$
\mathbf{H}(\vec{x})=\left(H^{0}, H^{\Lambda \Sigma} ; H_{0}, H_{\Lambda \Sigma}\right)=\mathbf{h}+\sum_{s=1}^{n} \frac{\boldsymbol{\Gamma}_{s}}{\left|\vec{x}-\vec{x}_{s}\right|},
$$

which gives the stationary metric for the BPS multicenter solution as

$$
d s_{4}^{2}=-J_{4}^{-1 / 2}(\vec{x})(d t+\vec{\omega} d \vec{x})^{2}+J_{4}^{1 / 2}(\vec{x}) d \vec{x}^{2} .
$$

The standard definitions still apply:

$$
\begin{gathered}
J_{4}(\vec{x}) \equiv J_{4} \circ \mathbf{H}(\vec{x}), \quad J_{4}(\mathbf{h})=1, \\
\nabla \times \vec{\omega}=\langle\mathbf{H}, \nabla \mathbf{H}\rangle,
\end{gathered}
$$

as well as the usual integrability condition:

$$
\sum_{t=1}^{n} \frac{\left\langle\boldsymbol{\Gamma}_{s}, \boldsymbol{\Gamma}_{t}\right\rangle}{\left|\vec{x}_{s}-\vec{x}_{t}\right|}+\left\langle\boldsymbol{\Gamma}_{s}, \mathbf{h}\right\rangle=0 .
$$

The vector fields are combined into a constrained complex matrix defined just as in Eq. (4.16). The 30 scalars of magic quaternionic model represent the coset space $\frac{S O^{*}(12)}{U(6)}$. We will give the solution for them using our modified attractor Eqs. (3.49)

$$
\frac{X^{\Lambda \Sigma}}{X^{0}}(\vec{x})=\frac{H^{\Lambda \Sigma}+2 i \frac{\partial \sqrt{J_{4}(H)}}{\partial H_{\Lambda \Sigma}}}{p^{0}+i \frac{\partial \sqrt{J_{4}(H)}}{\partial H_{0}}},
$$

also written as

$$
\frac{X_{1}^{\Lambda \Sigma}+i X_{2}^{\Lambda \Sigma}}{X^{0}}(\vec{x})=\frac{H_{1}^{\Lambda \Sigma}+i \frac{\partial \sqrt{J_{4}(H)}}{\partial H_{1 \Lambda \Sigma}}}{p^{0}+i \frac{\partial \sqrt{J_{4}(H)}}{\partial H_{0}}}+i \frac{H_{2}^{\Lambda \Sigma}+i \frac{\partial \sqrt{J_{4}(H)}}{\partial H_{2 \Lambda \Sigma}}}{p^{0}+i \frac{\partial \sqrt{J_{4}(H)}}{\partial H_{0}}} .
$$

At infinity the scalars take values defined by the harmonic function at infinity, when $\mathbf{H}=\mathbf{h}$. Near each center at $\vec{x}=$ $\vec{x}_{s}$ the attractor values of the scalars are defined as follows:

$$
\frac{X^{\Lambda \Sigma}}{X^{0}}\left(\vec{x}_{s}\right)=\left.\frac{H^{\Lambda \Sigma}+2 i \frac{\partial \sqrt{J_{4}(H)}}{\partial H_{1 \Lambda \Sigma}}}{p^{0}+i \frac{\partial \sqrt{J_{4}(H)}}{\partial H_{0}}}\right|_{H \Rightarrow \Gamma_{s} / \mid \vec{x}-\vec{x}_{s}} .
$$

Note that if we would keep only canonical $3+3$ of our 15 $p$ and $q$ we would reproduce the Caley's Hyperdeterminant of the $2 \times 2 \times 2$ matrix, which will also give us the STU multicenter black hole solutions.

\section{B. On the uniqueness of the $N=8$ multicenter BPS solution}

We want to argue that the most general $1 / 8$ BPS multicenter solution is given by the $1 / 2$ BPS solution of the quaternionic magic $N=2$ supergravity, modulo and overall $S U(8)$ rotation. Indeed, using an $S U(8)$ rotation one can diagonalize the $N=8$ central charge matrix so that it has only the nonvanishing complex eigenvalues $z_{1}=Z_{12}, z_{2}=$ $Z_{34}, z_{3}=Z_{56}, z_{4}=Z_{78}$. As argued in Ref. [9] this leads to a condition at each attractor point that

$$
\begin{gathered}
z_{1} z_{2}+z^{* 3} z^{* 4}=0, \quad z_{1} z_{3}+z^{* 2} z^{* 4}=0, \\
z_{2} z_{3}+z^{* 1} z^{* 4}=0 .
\end{gathered}
$$

The 1/8 BPS condition for the one center solution requires that

$$
\begin{gathered}
z_{1}=\rho_{\mathrm{BPS}} e^{i \varphi_{1}} \neq 0, \quad z_{2}=z_{3}=z_{4}=0, \\
J_{4}^{\mathrm{BPS}}=\rho_{\mathrm{BPS}}^{4}>0, \quad M_{\mathrm{BPS}}=\left|z_{1}\right| .
\end{gathered}
$$

The largest central charge $z_{1}$ belongs naturally to a unique $N=2$ subalgebra. This defines a decomposition of the $N=8$ supergravity. In the compact basis $S U(2) \times S U(6)$ 
under $S U(8)$ of $E_{7(7)}$

$$
\begin{aligned}
\mathbf{2 8} \text { of } S U(8) \rightarrow \mathbf{2 8}= & (\mathbf{1}, \mathbf{1 5})+(\mathbf{2}, \mathbf{6}) \\
& +(\mathbf{1}, \mathbf{1}) \text { of } S U(2) \times S U(6) .
\end{aligned}
$$

The $1+15$ are antisymmetric and make a total of $32(16$ electric and 16 magnetic). This is precisely like $28+28$ combine in 56 of $E_{7(7)}$. However, now the 32 combine into a single chiral spinor of $S O^{*}(12)$, which is the U-duality group of the truncated $N=2$ theory.

In general finding $1 / 8$ BPS solutions of $N=8$ supergravity, $1 / 6 \mathrm{BPS}$ solutions of $N=6$ supergravity, 1/4 BPS solutions to $N=4$ supergravity always involves a consistent truncation to the appropriate $N=2$ supergravity. For the case of $N=8$, this gives the quaternionic magic $N=2$ supergravity.

\section{Multicenter case}

It is plausible that for the solution to be BPS each center must be $1 / 2$ BPS with respect to the same $N=2$ algebra. In such a case, we may truncate our theory to a single copy of the magic supergravity with vector multiplets only and ignore all the hypermultiplets, as we did in the previous section, and we may claim that there are no other solutions. The question which one still may like to ask here is the following. Is it necessary that near each center the BPS solution can only belong to the same $N=2$ supergravity? Could it be that more general solution has different $N=2$ parts of $N=8$ as unbroken supersymmetry? For example, at some center instead of Eq. (5.15) we may have

$$
\begin{gathered}
z_{1}=z_{3}=z_{4}=0, \quad z_{2}=\rho_{\mathrm{BPS}} e^{i \varphi_{1}} \neq 0, \\
J_{4}^{\mathrm{BPS}}=\rho_{\mathrm{BPS}}^{4}>0, \quad M_{\mathrm{BPS}}=\left|z_{2}\right| .
\end{gathered}
$$

If this is possible, one would not be able to provide the most general $1 / 8$ BPS solution of $N=8$ by truncating it to quaternionic magic $N=2$. However, we find this scenario extremely unlikely.

One way to resolve this issue and get a definite statement on the uniqueness/nonuniqueness of our solution is by using the study of the Killing spinors of 1/8 BPS multicenter solutions in $N=8$ supergravity. These Killing spinors have not been constructed so far. We find it plausible to conjecture that such Killing spinors have the structure seen in many other examples, namely: the Killing spinor depends on $\vec{x}$ via a common factor $e^{U(\vec{x}) / 2}$, where the $g_{t t}=$ $e^{2 U(\vec{x})}$, and a phase. The Killing spinor also satisfies some constraint imposed globally. Thus the projector usually acts on a part of the spinor which is $\vec{x}$-independent. The reason is that the supersymmetry transformations [1] are given by

$$
\begin{gathered}
\delta \Psi_{\mu A}=D_{\mu} \epsilon_{A}+Z_{A B \mu \nu} \gamma^{\nu} \epsilon^{B}, \\
\delta \chi_{A B C}=\gamma^{\mu} P_{\mu A B C D} \epsilon^{D}+Z_{[A B \mu \nu} \sigma^{\mu \nu} \epsilon_{C]} .
\end{gathered}
$$

They consist of two parts, the first is the gravitino in Eq. (5.18) while the second is for the spin $1 / 2$ fields in Eq. (5.19). We deal only with the global part of the spinor for the spin $1 / 2$ equation while for the gravitino we have a spin connection which tells us how the Killing spinor acquires an $\vec{x}$-dependent factor, which depends on some harmonic functions. The projector, however, is the same for all centers if the Killing spinor in $N=8$ has the same features as the one in truncated models. If our expectation about the structure of Killing spinors are justified, we will be able to confirm definitely the uniqueness of our solution for $1 / 8$ BPS multicenter black holes in $N=8$ supergravity.

\section{Alternate $N=8$ structure and the black hole mass formula}

In the material above, we have written the gauge fields, prepotential and complex scalars using a symplectic section nicely adapted to an invariance under the fivedimensional U-duality group $G_{5}$. We will not give any description of the gauge fields or the coset which is explicitly invariant under $G_{4}$, but is useful to point out such a description is easily available for understanding the metric. This description uses some of the inherent structures of the $N=8$ U-duality group, and by truncation applies to the quaternionic magic $N=2$. It is likely that the modification of a reality structure yields a similar structure for the octonionic magic $N=2$, but we will not explore this here.

The $N=8$ U-duality group in four dimensions, $G_{4}=$ $E_{7(7)}$ has a covariant cubic map [35] from the the fundamental representation back into itself and an invariant symplectic form. Each $56\left(X^{i j}, X_{i j}\right)$, is spanned by the two antisymmetric real tensors $X^{i j}$ and $X_{i j}$ and the action of $E_{7(7)}$ on them is realized in a standard way [1,35].

The quadratic invariant of $E_{7(7)}$, an invariant symplectic form, can be constructed from any two distinct fundamentals as follows.

$$
\langle X, Y\rangle=-\langle Y, X\rangle:=X^{i j} Y_{i j}-X_{i j} Y^{i j}
$$

Note that such quadratic invariant vanishes for a single fundamental. A triple product of any three $\mathbf{5 6}\left((X, Y, Z)^{i j}\right.$, $\left.(X, Y, Z)_{i j}\right)$, gives a trilinear map $\mathbf{5 6} \times \mathbf{5 6} \times \mathbf{5 6} \rightarrow \mathbf{5 6}$.

A unique quartic invariant can be constructed from a single fundamental in the following way. One can build out of $X$ a triple which is different from the original 56. Afterwards one can use the quadratic invariant for 2 distinct fundamentals: a fundamental and its triple: this gives the standard form of a quartic invariant via the following symplectic invariant

$$
J_{4}(X)=\frac{1}{48}\langle(X, X, X), X\rangle .
$$

The triple product obeys some relations 


$$
\begin{aligned}
& (X, Y, Z)=(Y, X, Z)+2\langle X, Y\rangle Z, \\
& (X, Y, Z)=(Z, Y, X)-2\langle X, Z\rangle Y .
\end{aligned}
$$

A symplectic product of a fundamental and a triple satisfies the following relation

$$
\langle(X, Y, Z), W\rangle=\langle(X, W, Z), Y\rangle-2\langle X, Z\rangle\langle Y, W\rangle .
$$

The operations above have their origin in the Jordan algebra structure, $J_{3}^{\mathbb{Q}_{s}}$ behind the magic square construction of $E_{7}$, and so carry over nicely to the duality group based on the restriction of this algebra to $J_{3}^{\mathbb{Q}}$, the quaternionic Uduality group $G_{4}=S O^{*}(12)$. This allows us to write for all magic supergravities:

$$
J_{4}(\vec{x})=\frac{1}{48}\langle(\mathbf{H}(\vec{x}), \mathbf{H}(\vec{x}), \mathbf{H}(\vec{x})), \mathbf{H}(\vec{x})\rangle .
$$

Here the corresponding harmonic function $\mathbf{H}(\vec{x})$ is in the fundamental representation of $E_{7(-25)}$ for octonionic magic and in the appropriate representation for all its truncations. In particular, in the quaternionic case this expression provides us with the mass of the $N=8$ supergravity solution. We can efficiently extract this mass for our solution using the form for $J_{4}$ above, $J_{4}$ 's fall-off at infinity, the properties of the cubic map and the fact that $\langle\mathbf{h}, \boldsymbol{\Gamma}\rangle=0$. The ADM mass is defined via the asymptotic behavior of the timetime component of the metric, as

$$
g_{t t}=J_{4}^{-1 / 2}=1-\frac{2 M_{\mathrm{ADM}}}{|\vec{x}|}+\ldots
$$

and we take into account that in our case

$$
\begin{aligned}
\langle(\mathbf{h}, \mathbf{h}, \mathbf{h}), \boldsymbol{\Gamma}\rangle & =\langle(\boldsymbol{\Gamma}, \mathbf{h}, \mathbf{h}), \mathbf{h}\rangle=\langle(\mathbf{h}, \boldsymbol{\Gamma}, \mathbf{h}), \mathbf{h}\rangle \\
& =\langle(\mathbf{h}, \mathbf{h}, \boldsymbol{\Gamma}), \mathbf{h}\rangle .
\end{aligned}
$$

This gives us a magic black hole mass formula:

$$
M_{\mathrm{ADM}}=\frac{1}{48}\langle(\mathbf{h}, \mathbf{h}, \mathbf{h}), \boldsymbol{\Gamma}\rangle .
$$

\section{BPS AND NON-BPS, $N=2 \operatorname{VERSUS~} N=5,6,8$}

\section{A. $N=\mathbf{2}$ attractors, general case}

In [9] simple algebraic attractor equations were derived for regular black holes of $N=8$ supergravity. These equations have 2 solutions: one BPS with $1 / 8$ of unbroken supersymmetry and one non-BPS solution with all supersymmetries broken. In the case of the STU truncation the $N=8$ attractor equations correspond to the attractor equations of the $N=2$ model.

Here we would like to analyze the generic attractor equations for $N=2$ theory and apply this analysis to BPS and non-BPS attractors of magic supergravities. For this purpose we start with a special geometry identity:

$$
\begin{aligned}
& p^{\Sigma}+i \frac{\partial I_{1}}{\partial q_{\Sigma}}=2 i \bar{Z} L^{\Sigma}+2 i G^{j \bar{j}} \mathcal{D}_{j} Z \mathcal{D}_{\bar{j}} \bar{L}^{\Sigma}, \\
& q_{\Sigma}-i \frac{\partial I_{1}}{\partial p^{\Sigma}}=2 i \bar{Z} M_{\Sigma}+2 i G^{j \bar{j}} \mathcal{D}_{j} Z \mathcal{D}_{\bar{j}} \bar{M}_{\Sigma} .
\end{aligned}
$$

The identity follows from the definition

$$
\mathcal{D}_{j} Z=\left(\partial_{j}+\frac{1}{2} K_{, j}\right) Z,
$$

where the central charge $Z=L^{\Lambda} q_{\Lambda}-M_{\Lambda} p^{\Lambda}$. One contracts Eq. (6.2) with $\mathcal{D}_{\bar{j}} \bar{L}^{\Sigma} G^{\bar{j} i}$ and proceed as in Eqs. (40)(45) in Ref. [6] where the BPS attractor equations were derived assuming $D Z=0$. A simplified form of these equations, which uses the derivatives of the $I_{1}(p, q)$-invariant, was explained in Sec. 3.2 in Ref. [36]. Eqs. (6.1) supplemented by a vanishing of the first derivative of the potential, $2 \bar{Z} \mathcal{D}_{i} Z+i c_{i j k} G^{i \bar{j}} G^{k \bar{k}} \mathcal{D}_{\bar{j}} \bar{Z} \mathcal{D}_{\bar{k}} \bar{Z}=0$ [8], have 3 types of solutions.

(1) $1 / 2$ BPS, well known

$$
p^{\Sigma}+i \frac{\partial I_{1}}{\partial q_{\Sigma}}=2 i \bar{Z} L^{\Sigma}, \quad q_{\Sigma}-i \frac{\partial I_{1}}{\partial p^{\Sigma}}=2 i \bar{Z} M_{\Sigma} .
$$

(2) Non-BPS, $Z=0$, less known

$$
\begin{aligned}
& p^{\Sigma}+i \frac{\partial I_{1}}{\partial q_{\Sigma}}=2 i G^{j \bar{j}} \mathcal{D}_{j} Z \mathcal{D}_{\bar{j}} \bar{L}^{\Sigma} \\
& q_{\Sigma}-i \frac{\partial I_{1}}{\partial p^{\Sigma}}=2 i G^{j \bar{j}} \mathcal{D}_{j} Z \mathcal{D}_{\bar{j}} \bar{M}_{\Sigma} .
\end{aligned}
$$

If $2 i G^{j \bar{j}} \mathcal{D}_{j} Z \mathcal{D}_{\bar{j}} \bar{L}^{\Sigma}=2 i \sum_{I=2}^{4} \bar{Z}_{I} \mathcal{D}_{\bar{I}} \bar{L}^{\Sigma}$ we find a solution of the following type

$$
Z_{2} \neq 0, \quad Z_{3}=0, \quad Z_{4}=0,
$$

and we can solve the attractor equations as follows:

$$
\frac{p^{\Lambda}+i \frac{\partial I_{1}}{\partial q_{\Lambda}}}{p^{0}+i \frac{\partial I_{1}}{\partial q_{0}}}=\frac{\overline{\mathcal{D}}_{\hat{2}} \bar{L}^{\Lambda}}{\overline{\mathcal{D}}_{\hat{2}} \bar{L}^{0}}, \quad \frac{q_{\Lambda}-i \frac{\partial I_{1}}{\partial p^{\Lambda}}}{q_{0}-i \frac{\partial I_{1}}{\partial p^{0}}}=\frac{\overline{\mathcal{D}}_{\hat{2}} \bar{M}_{\Lambda}}{\overline{\mathcal{D}}_{\hat{2}} \bar{M}_{0}} .
$$

(3) Non-BPS, $Z \neq 0$. This case is particularly tractable for the models with the coset spaces $\frac{G_{4}}{H_{4}}$ of rank 3. All magic models have rank 3 . The matrix of central charge derivatives $D Z$ has an $H_{4}$ symmetry:

(i) $J^{\mathbb{Q}} \Rightarrow H_{4}=E_{6} \times U(1)$,

(ii) $J^{\mathbb{Q}} \Rightarrow H_{4}=S U(6) \times U(1)$,

(iii) $J^{\mathbb{C}} \Rightarrow H_{4}=S U(3) \times S U(3) \times U(1)$,

(iv) $J^{\mathbb{R}} \Rightarrow H_{4}=S U(3) \times U(1)$.

With an $H_{4}$ rotation we can skew-diagonalize the matrix $D Z$ so that it has 3 eigenvalues, $\overline{\mathcal{D}}_{I} \bar{Z} \equiv Z_{I}, I=2,3,4$. Together with $N=2$ central charge $Z=-i Z_{1}$ we have 4 charges, 


$$
Z_{1}=i Z, \quad Z_{I}=\bar{D}_{\hat{I}} \bar{Z} .
$$

The extremality condition is given by

$$
2 \bar{Z} \mathcal{D}_{\hat{I}} Z+i d_{\hat{I} \hat{J} \hat{K}} \bar{Z}_{\hat{J}} \bar{Z}_{\hat{K}} \delta^{\hat{J} \hat{J}} \delta^{\hat{K} \hat{K}}=0,
$$

and it becomes for magic models in normal frame

$$
\begin{gathered}
Z_{1} Z_{2}+Z^{* 3} Z^{* 4}=0, \quad Z_{1} Z_{3}+Z^{* 2} Z^{* 4}=0, \\
Z_{2} Z_{3}+Z^{* 1} Z^{* 4}=0,
\end{gathered}
$$

exactly as in $N=8$ case [9]. The solution is as in Ref. [9]

$$
\begin{aligned}
& Z_{1}=\rho e^{i(\pi-3 \phi)}, \quad Z_{I}=\rho e^{i \phi}, \\
& Z_{1} Z_{2} Z_{3} Z_{4}=\rho^{4} e^{i \pi}=-\rho^{4} .
\end{aligned}
$$

Here we have satisfied the attractor equations: $Z_{I} Z_{J}+$ $Z_{K}^{*} Z_{M}^{*}=0, I \neq J \neq K \neq M$. We may use this in an identity (6.1) and we find in case that $\phi=0$

$$
\begin{gathered}
p^{\Sigma}+i \frac{\partial I_{1}}{\partial q_{\Sigma}}=2 \rho\left(L^{\Sigma}+i \sum_{\hat{I}} \overline{\mathcal{D}}_{\hat{I}} \bar{L}^{\Sigma}\right), \\
q_{\Sigma}-i \frac{\partial I_{1}}{\partial p^{\Sigma}}=2 \rho\left(M_{\Sigma}+i \sum_{\hat{I}} \overline{\mathcal{D}}_{\hat{\bar{I}}} \bar{M}_{\Sigma}\right),
\end{gathered}
$$

and

$$
\begin{gathered}
\frac{p^{\Sigma}+i \frac{\partial I_{1}}{\partial q_{\Sigma}}}{p^{0}+i \frac{\partial I_{1}}{\partial q_{0}}}=\frac{L^{\Sigma}+i \sum_{\hat{I}} \overline{\mathcal{D}}_{\hat{I}} \bar{L}^{\Sigma}}{L^{0}+i \sum_{\hat{I}} \overline{\mathcal{D}}_{\hat{I}} \bar{L}^{0}}, \\
\frac{q_{\Sigma}-i \frac{\partial I_{1}}{\partial p^{\Sigma}}}{q_{0}-i \frac{\partial I_{1}}{\partial p^{0}}}=\frac{M_{\Sigma}+i \sum_{\hat{I}} \overline{\mathcal{D}}_{\hat{I}} \bar{M}_{\Sigma}}{M_{0}+i \sum_{\hat{I}} \overline{\mathcal{D}}_{\hat{I}} \bar{M}_{0}} .
\end{gathered}
$$

Eqs. (6.13) define the values of moduli fields in terms of charges in the non-BPS case. We have verified [37] that these equations provide a non-BPS attractor solution of the STU model in example studied in Ref. [11].

\section{B. $N=\mathbf{2}$ versus $N=5,6,8$ and BPS versus, non-BPS}

The extended supergravities $N=5,6$ as well as $N=8$ have no matter multiplets, only the gravitational one. Therefore they are very restricted. BPS black holes in these theories have been studied before in Ref. [38]. Since now we understand certain features on $N=8$ BPS and nonBPS black holes and their relation to $N=2$ BPS and nonBPS ones, as shown in Ref. [9], we may extend this relations also to include $N=6$ and $N=5$ theories.

The $N=8$ supergravity has a consistent truncation both to $N=6$ and $N=2$ supergravity theories, depending on whether one keeps six or two of the eight gravitinos. In both cases one ends up with $J^{Q}$ based on the $\frac{S O^{*}(12)}{U(6)}$ mani- fold. This manifold, because it is consistent with $N=2$, is indeed a special Kähler symmetric space. The charge vector is in the 32 of $S O^{*}(12)$ (chiral spinor). From an $N=$ 2 point of view

$$
32=15+\overline{\mathbf{1 5}}+\mathbf{1}+\overline{\mathbf{1}},
$$

where $\mathbf{1 5}$ are in the matter multiplet and $\mathbf{1}$ is the graviphoton.

In $N=6$ supergravity the central charge is $Z^{A B}=$ $-Z^{B A}$ with $A, B=1, \ldots, 6$ and there is also a singlet charge $Z$ [38]. The black hole potential is

$$
V_{\mathrm{BH}}=\frac{1}{2} Z_{A B} \bar{Z}^{A B}+Z \bar{Z} \text {. }
$$

The $1 / 6$ BPS solution of $N=6$ theory was identified in Ref. [38]: it has $Z=0$ and a skew-diagonal $Z_{A B}$ with $Z_{12} \neq 0$ and $Z_{34}=Z_{56}=0$. From the $N=2$ point of view such solution is a non-BPS solution (with vanishing central charge). On the other hand, the $1 / 2$ BPS solution of $N=2$ theory with $Z \neq 0$ and $Z_{A B}=0$ corresponds to a non-BPS solution of the $N=6$ theory (with vanishing $N=6$ central charge).

Let us now comment on $N=5$ theory and relation of $1 / 5 \mathrm{BPS}$ solution to $1 / 2 \mathrm{BPS}$ of the relevant $N=2$ theory. The $N=5$ special Kähler geometry theory is based on the $\frac{S U(5,1)}{U(5)}$ symmetric space with 5 complex scalars. The central charge $Z_{A B}$ has a nonvanishing component $Z_{12} \neq 0$ whereas $Z_{34}=0$ is vanishing: this is a $1 / 5$ BPS solution. Despite the fact that the $N=5$ model has the same sigma model as the corresponding $N=2$ theory, the vector part of these models is different: $N=2$ has six vectors and $N=5$ has ten vectors. Therefore, as different from $N=6$ case, the $1 / 5$ BPS solutions of $N=5$ are different from the $1 / 2$ of $N=2$ supergravity based on $\frac{S U(5,1)}{U(5)}$ symmetric space.

\section{DISCUSSION}

We have given a complete description of the cubic and quartic invariants for magic supergravities. Using these we were able to write down multicenter 1/2 BPS solutions for the exceptional octonionic magic supergravity with a 56component charge vector at each center. This model has $E_{7(-25)}$ duality symmetry, which is slightly different from the duality symmetry of $N=8$ theory, $E_{7(7)}$. We have also demonstrated how multicenter quaternionic magic solutions with 32-component charge at each center can be used to describe a broad (possibly complete) array of $N=$ 8 1/8 BPS multicenter solution.

In Ref. [28], it was shown that any single center $1 / 8$ BPS solution of $N=8$ could be rotated using an $E_{7(7)}$ duality transformation to a form involving just a single center solution the $N=2$ STU truncation of $N=8$. For the non-BPS attractors of magic supergravities we have made used of this truncation to the STU model. Using the solutions of the non-BPS attractors for $N=8$ super- 
gravity presented in [9] we have found the general solutions of the non-BPS attractors for the $N=2$ STU model.

The duality rotation which takes a single center solution to an STU form is not powerful enough for a generic $1 / 8$ BPS solution with multiple centers: such a rotation is clearly not enough to rotate every center to STU form. For solutions generated via quaternionic magic supergravity, it would be interesting to work out which is the minimal $N=2$ truncation that admits generic cases with two, three, four etc... centers, just as STU supergravity is minimal truncation of $N=8$ which describes all single center solutions.

Clearly, $N=2$ truncations play a major role in understanding 1/8 BPS states of $N=8$ supergravity. Apart from clarifying if $N=2$ truncation is the only way to achieve $1 / 8$ BPS configurations, a clearer look at these truncations in context of black holes might be productive. Different truncations allow a varying number of hyper-multiplet scalars (see e.g. Ref. [39]) from the $N=8$ coset to survive. The hyper scalars vevs typically play a spectator role, since they will not vary in BPS black hole solutions, but for an observer near infinity they may reveal some information about just which $N=2$ truncation is in operation for a given multicenter solution.

For future reference, we summarize the hyper-multiplet scalars which the survive from the $N=8$ coset in the truncation to the various magic supergravities (for details see Ref. [39]). In quaternionic magic, we have $E_{7(7)} \rightarrow$ $S O^{*}(12) \times S U(2)$ and no hypers survive. In the complex case, $E_{7(7)} \rightarrow S U(3,3) \times S U(2,1)$ and we get four hyper scalars parameterizing the coset $S U(2,1) / S U(2) \times U(1)$; the real case corresponds to $E_{7(7)} \rightarrow S p(6, \mathbb{R}) \times G_{2(2)}$ and has eight hyper scalars, $G_{2(2)} / S O(4)$. The STU truncations has hyper scalars in $S O(4,4) / S O(4) \times S O(4,4)$ (see Ref. [28]). As we restrict the vector moduli space, the hyper moduli space increases in multiples of four (it is quaternionic).

Finally, given the relationship between extremal nonBPS and BPS solutions of $N=2$ magic quaternionic supergravity and those of $N=6$, it would be interesting to understand just how many such links exists between various extremal solutions of different supergravities, perhaps along lines related to Ref. [36].

\section{ACKNOWLEDGMENTS}

It is a pleasure to thank R. D'Auria, V. Balasubramanian, O. Ganor, M. Günaydin, T. Levi, T. Ortín, B. Pioline, A. Sen, A. Tomasiello, and M. Trigiante for useful conversations. We are grateful to participants of 2006 Frascati Winter school on Attractor mechanism for the interest to this work. The work of S. F. has been supported in part by the European Community Human Potential Program under Contract No. MRTN-CT-2004-005104 "Constituents, fundamental forces and symmetries of the universe," in association with INFN Frascati National Laboratories and by
D.O.E. Grant No. DE-FG03-91ER40662, Task C. The work of E. G. was supported by the US Department of Energy under Contracts No. DE-AC03-76SF00098 and No. DE-FG03-91ER-40676 and by the National Science Foundation under Grant No. PHY-00-98840. The work of R. K. was supported by NSF Grant No. PHY-0244728.

\section{APPENDIX: $d=6$ DUALITY AND QUATERNIONIC MAGIC MODEL}

This model even if we break the manifest $G_{5}$ duality down to $G_{6}$ duality still does not give us a very special real geometry in $d=5$ and the relevant complexified special geometry in $d=4$. The reason is that the spinors of $S O(1,5) \sim S U^{*}(4)$ as opposite to other magic models with $S O(1,2), S O(1,3), S O(1,9)$ do not have real representations. Thus we have to use complex coordinates constrained by reality conditions.

Both the vectors and the spinors are complex and satisfy the reality condition. For vectors of $S U^{*}(4)$ we use an antisymmetric matrix $V_{A B}=-V_{B A}$ and $V^{*}=-\Omega V \Omega$ where $\quad \Omega=-\Omega^{t}, \quad \Omega^{2}=-\mathbf{1}$. Thus $V_{1}=\operatorname{Re} V=$ $-\Omega \operatorname{Re} V \Omega$ and $V_{2}=\operatorname{Im} V=\Omega \operatorname{Im} V \Omega$. We also introduce $\tilde{V}^{A B} \equiv \frac{1}{2} \epsilon^{A B C D} V_{C D}$. Thus the first term in the cubic invariant, $z\left[\left(b^{0}\right)^{2}-\sum_{i=1}^{i=5}\left(b^{i}\right)^{2}\right]$ can be rewritten as $z V_{A B} \tilde{V}^{A B}=$ $z\left(V_{1 A B} \tilde{V}_{1}^{A B}-V_{2 A B} \tilde{V}_{2}^{A B}\right)$ in terms of real entries only. For spinors $\Psi_{A i}$ of $S U^{*}(4) \times S U(2)$ with $A=1, \ldots, 4, i=1,2$ there is a reality condition $\Psi_{A i}^{*}=\Omega_{A B} \epsilon_{i j} \Psi_{B j}$ and $\epsilon^{2}=$ -1 . We also split these spinors into real and imaginary parts which we call $\Psi_{1}$ and $\Psi_{2}$ respectively, $\Psi_{1}=\Omega \epsilon \Psi_{1}$ and $\Psi_{2}=-\Omega \epsilon \Psi_{2}$. The second term of the cubic invariant can now be presented as a function of only real coordinates, $\quad\left[V_{1 A B}\left(\Psi_{1}^{A i} \Psi_{1}^{B j}-\Psi_{2}^{A i} \Psi_{2}^{B j}\right)-V_{2 A B}\left(\Psi_{1}^{A i} \Psi_{2}^{B j}+\right.\right.$ $\left.\left.\Psi_{1}^{B j} \Psi_{2}^{A i}\right)\right] \epsilon_{i j}$.

Now we need the cubic invariants in terms of charges. We take

$$
\begin{gathered}
p^{z}, p^{r}, p^{\alpha} \Rightarrow p^{z}, p_{A B}, p^{A i}, \quad q_{z}, q_{r}, q_{\alpha} \Rightarrow q_{z}, q^{A B}, q_{A i}, \\
p_{A B}=-p_{B A}, \quad q^{A B}=-q^{B A}, \\
\text { and } \quad p \cdot q=p^{z} q_{z}+p_{A B} q^{A B}+p^{A i} q_{A i}, \\
\frac{1}{2} \epsilon^{A B C D} p_{C D}, \quad \tilde{q}_{A B}=\frac{1}{2} \epsilon_{A B C D} q^{C D} .
\end{gathered}
$$

The cubic invariants are

$$
\begin{gathered}
I_{3}(p)=\frac{1}{2}\left(p^{z} p_{A B} \tilde{p}^{A B}+p_{A B} p^{A i} p^{B j} \epsilon_{i j}\right), \\
I_{3}(p)=\frac{1}{2}\left(q_{z} q^{A B} \tilde{q}_{A B}+q^{A B} q_{A i} q_{B j} \epsilon^{i j}\right),
\end{gathered}
$$

where the reality conditions are

$$
p^{*}=-\Omega p \Omega, \quad p^{* A i}=(\Omega \epsilon p)^{A i} .
$$

As the consequence of the reality conditions (A4) the cubic invariants are real

$$
I_{3}^{*}(p)=I_{3}\left(p^{*}\right)=I_{3}(p)
$$

and the same for electric charges. We may split the charges 
into real and imaginary parts

$$
p_{A B}=p_{1 A B}+i p_{2 A B}, \quad p_{1}^{A i}+i p_{2}^{A i},
$$

and find for $I_{3}(p)$

$$
\begin{gathered}
\frac{1}{2}\left(p^{z}\left(p_{1 A B} \tilde{p}_{1}^{A B}-p_{2 A B} \tilde{p}_{2}^{A B}\right)+\left[p_{1 A B}\left(p_{1}^{A i} p_{1}^{B j}-p_{2}^{A i} p_{2}^{B j}\right)\right.\right. \\
\left.\left.-p_{2 A B}\left(p_{1}^{A i} p_{2}^{B j}+p_{2}^{A i} p_{1}^{B j}\right)\right] \epsilon_{i j}\right),
\end{gathered}
$$

and analogous for $I_{3}(q)$.
[1] E. Cremmer and B. Julia, Nucl. Phys. B159, 141 (1979); B. de Wit and H. Nicolai, Nucl. Phys. B208, 323 (1982).

[2] M. Gunaydin, G. Sierra, and P. K. Townsend, Phys. Lett. B 133, 72 (1983); Nucl. Phys. B242, 244 (1984); B253, 573 (1985).

[3] S. Ferrara and M. Gunaydin, Int. J. Mod. Phys. A 13, 2075 (1998).

[4] H. Freudenthal, Proc. Konink. Ned. Akad. Wetenschap A 62, 447 (1959); B. A. Rozenfeld, Doklady Seriia biologiia/ Akademii nauk SSSR 106, 600 (1956); J. Tits, Mem. Acad. Roy. Belg. Sci. 29, 3 (1955).

[5] S. Ferrara, R. Kallosh, and A. Strominger, Phys. Rev. D 52, R5412 (1995); A. Strominger, Phys. Lett. B 383, 39 (1996).

[6] S. Ferrara and R. Kallosh, Phys. Rev. D 54, 1514 (1996).

[7] S. Ferrara and R. Kallosh, Phys. Rev. D 54, 1525 (1996).

[8] S. Ferrara, G. W. Gibbons, and R. Kallosh, Nucl. Phys. B500, 75 (1997).

[9] S. Ferrara and R. Kallosh, Phys. Rev. D 73, 125005 (2006).

[10] R. R. Khuri and T. Ortin, Phys. Lett. B 373, 56 (1996); T. Ortin, Phys. Lett. B 422, 93 (1998); hep-th/9705095.

[11] P. K. Tripathy and S. P. Trivedi, J. High Energy Phys. 03 (2006) 022; R. Kallosh, N. Sivanandam, and M. Soroush, J. High Energy Phys. 03 (2006) 060; S. Bellucci, S. Ferrara, and A. Marrani, Phys. Lett. B 635, 172 (2006).

[12] B. Bates and F. Denef, hep-th/0304094; F. Denef, hep-th/ 0010222; J. High Energy Phys. 08 (2000) 050.

[13] A. Ceresole, R. D'Auria, and S. Ferrara, Nucl. Phys. B, Proc. Suppl. 46, 67 (1996).

[14] K. Behrndt, D. Lust, and W. A. Sabra, Nucl. Phys. B510, 264 (1998); G. Lopes Cardoso, B. de Wit, J. Kappeli, and T. Mohaupt, J. High Energy Phys. 12 (2000) 019.

[15] E. Bergshoeff, R. Kallosh, and T. Ortin, Nucl. Phys. B478, 156 (1996).

[16] V. Balasubramanian, E. G. Gimon, and T. S. Levi, hep-th/ 0606118.

[17] E. Cremmer and A. Van Proeyen, Classical Quantum Gravity 2, 445 (1985).

[18] B. de Wit and A. Van Proeyen, Commun. Math. Phys. 149, 307 (1992).

[19] B. de Wit, F. Vanderseypen, and A. Van Proeyen, Nucl. Phys. B400, 463 (1993).

[20] K. Behrndt, R. Kallosh, J. Rahmfeld, M. Shmakova, and W. K. Wong, Phys. Rev. D 54, 6293 (1996).
[21] R. Kallosh, M. Shmakova, and W. K. Wong, Phys. Rev. D 54, 6284 (1996).

[22] K. Behrndt and W. A. Sabra, Phys. Lett. B 401, 258 (1997).

[23] D. Shih, A. Strominger, and X. Yin, J. High Energy Phys. 06 (2006) 037; hep-th/0505094; D. Gaiotto, A. Strominger, and X. Yin, J. High Energy Phys. 02 (2006) 024.

[24] B. Pioline, J. High Energy Phys. 08 (2005) 071.

[25] S. Helgason, Differential Geometry, Lie Groups, and Symmetric Spaces (Academic Press, New York, 1978).

[26] R. Gilmore, Lie Groups, Lie Algebras, and Some of Their Applications (Dover Publications, New York, 2006).

[27] R. Kallosh and B. Kol, Phys. Rev. D 53, R5344 (1996); M. Cvetic and C. M. Hull, Nucl. Phys. B480, 296 (1996); V. Balasubramanian, F. Larsen, and R. G. Leigh, Phys. Rev. D 57, 3509 (1998); M. Bertolini and M. Trigiante, Nucl. Phys. B582, 393 (2000).

[28] L. Andrianopoli, R. D'Auria, S. Ferrara, P. Fre, and M. Trigiante, Nucl. Phys. B509, 463 (1998).

[29] M. Gunaydin and O. Pavlyk, J. High Energy Phys. 01 (2005) 019.

[30] L. Andrianopoli, R. D'Auria, and S. Ferrara, Phys. Lett. B 403, 12 (1997).

[31] H. Ooguri, A. Strominger, and C. Vafa, Phys. Rev. D 70, 106007 (2004); V. Pestun, J. High Energy Phys. 09 (2006) 034; G. Lopes Cardoso, B. de Wit, J. Kappeli, and T. Mohaupt, J. High Energy Phys. 03 (2006) 074; J. P. Hsu, A. Maloney, and A. Tomasiello, J. High Energy Phys. 09 (2006) 048.

[32] A. Keurentjes, Nucl. Phys. B658, 303 (2003); B658, 348 (2003); Classical Quantum Gravity 20, S525 (2003).

[33] L. Andrianopoli, S. Ferrara, and M. A. Lledo, J. High Energy Phys. 06 (2004) 018.

[34] M. Gunaydin and O. Pavlyk, J. High Energy Phys. 08 (2005) 101.

[35] M. Gunaydin, K. Koepsell, and H. Nicolai, Commun. Math. Phys. 221, 57 (2001).

[36] R. Kallosh, hep-th/0603003.

[37] R. Kallosh, N. Sivanandam, and M. Soroush, Phys. Rev. D 74, 065008 (2006).

[38] L. Andrianopoli, R. D'Auria, and S. Ferrara, Int. J. Mod. Phys. A 13, 431 (1998); Phys. Lett. B 403, 12 (1997).

[39] L. Andrianopoli, R. D'Auria, and S. Ferrara, J. High Energy Phys. 03 (2002) 025. 